\title{
Die Sportschiedsgerichtsbarkeit im Lichte des Art. 6 EMRK
}

\author{
Florian Jung*
}

Inhalt

A. Wechselwirkungen von Sportschiedsgerichtsbarkeit und Art. 6 EMRK 173

B. Das Sportschiedsgericht als Gericht im Sinne des Art. 6 Abs. 1 EMRK 175

C. Bindung der Schiedsgerichte an Art. 6 Abs. 1 EMRK 178

$\begin{array}{ll}\text { I. Nationale Gerichtsentscheidungen } & 179\end{array}$

II. Rechtsauffassung der Rechtsprechungsorgane der EMRK 183

III. Meinungsstand in der Literatur 186

IV. Stellungnahme - Zugleich ein Plädoyer für eine unmittelbare Bindung
an Art. 6 Abs. 1 EMRK

1. Der Schiedsrichter - ein funktionaler Hoheitsträger im Lichte der EMRK 192

2. Sportspezifische Argumente 195

3. Zwischenergebnis 196

D. Umfang der Bindung an Art. 6 Abs. 1 EMRK 196

I. Verfahrensgarantien des Art. 6 Abs. 1 EMRK 196

II. Verzicht auf die Verfahrensgrundrechte des Art. 6 Abs. 1 EMRK 197

1. Zur Praxis: Schiedsvereinbarungen im Sportrecht 201

2. Rechtsprechungsübersicht 202

3. Stellungnahme 205

$\begin{array}{lll}\text { E. Fazit } 208 & 205\end{array}$

\section{A. Wechselwirkungen von Sportschiedsgerichtsbarkeit und Art. 6 EMRK}

Das Verhältnis des Sportschiedsgerichts zur Europäischen Menschenrechtskonvention (EMRK) hat bislang nur wenig Aufmerksamkeit in Wissenschaft und Praxis gefunden, womit die Einordnung der Sportschiedsgerichtsbarkeit in das Rechtssystem der EMRK

* Florian Jung, LL.M. (Eur.) ist wissenschaftliche Hilfskraft am Lehrstuhl für deutsches und ausländisches öffentliches Recht, Völkerrecht und Europarecht bei Prof. Dr. Stefanie Schmahl, LL.M. an der Universität Würzburg und Rechtsreferendar im Gerichtsbezirk des OLG Frankfurt am Main. Der Beitrag ist eine aktualisierte und überarbeitete Fassung der Magisterarbeit, die der Autor im Rahmen des Aufbaustudiums Europarecht an der Universität Würzburg angefertigt hat. Für wertvolle Hinweise und Anregungen sei Frau Prof. Schmahl herzlich gedankt. 
noch weitestgehend ein Schattendasein fristet. Eine Auseinandersetzung mit dieser Frage ist aber angesichts einer Vielzahl unterschiedlicher Entwicklungen im Sportrecht dringend angezeigt.

So haben Sportler in der jüngeren Vergangenheit vermehrt den Wunsch geäußert, den Europäischen Gerichtshof für Menschenrechte (EGMR) anzurufen, um vorangegangene Entscheidungen des Internationalen Sportgerichtshofs (Court of Arbitration for Sports, CAS) oder des Schweizer Bundesgerichts (BG) überprüfen zu lassen. Die Gründe für ein solches Anliegen lassen sich insbesondere anhand der negativen Konsequenzen einer positiven Dopingprobe treffend veranschaulichen, wie ein aktuelles Beispiel von den Olympischen Spielen in Sotschi zeigt. Der dort des Dopings beschuldigten (deutschen) Athletin droht etwa nicht nur die Aberkennung ihrer erzielten Ergebnisse, sondern auch eine langjährige Wettkampfsperre, die Aufkündigung von Sponsorenverträgen als auch die Herausnahme aus dem Spitzenförderungsprogramm der Bundeswehr. Angesichts dieser vielschichtigen Konsequenzen ist es nur allzu legitim, wenn die betroffenen Sportler alle (rechtlichen) Möglichkeiten ausschöpfen, gegen die (gegebenenfalls in ihren Augen ungerechtfertigte) positive Dopingprobe vorzugehen. Nachdem verschiedene Sportler, so etwa der spanische Radprofi Valverde, ${ }^{1}$ den Gang nach Straßburg zunächst allerdings nur angekündigt, nicht aber tatsächlich beschritten haben, beließ es die deutsche Eisschnellläuferin Claudia Pechstein nicht bei einer Ankündigung. Nachdem sowohl der CAS als auch das Schweizer Bundesgericht eine von der International Skating Union (ISU) verhängte Dopingsperre aufrechterhalten hatten, legte Pechstein Individualbeschwerde gegen die Schweiz vor dem EGMR wegen der Verletzung von Art. 6 EMRK ein, wo das Verfahren bis jetzt anhängig, ${ }^{2}$ aber noch nicht entschieden ist.

Auch haben prominente Athleten unmittelbar vor den Olympischen Spielen in Sotschi eine von Claudia Pechstein initiierte Petition unterzeichnet, worin das geltende System im internationalen Sportrecht erheblich kritisiert wird. ${ }^{3}$ Insbesondere die gängige Praxis der Unterzeichnung einer Athletenvereinbarung, wonach die Sportler die Zuständigkeit des CAS als letzte Instanz in Streit- und Dopingfällen anerkennen, ist nach Ansicht der Sportler problematisch, da sie durch die Unterzeichnung auf ihr Grundrecht verzichten, selbst in existenziellen Fragen ein (deutsches) Gericht anrufen zu können. Gleichzeitig sind sie gezwungen, diese Vereinbarung zu unterzeichnen, da ansonsten eine Teilnahme an großen Wettkämpfen, wie etwa den Olympischen Spielen, nicht möglich ist. Schützenhilfe hat diese Bewegung in einem aktuellen Judikat des Landgerichts München $\mathrm{I}^{4}$ erhalten. Das Gericht hat in diesem aufsehenerregenden Fall, der durchaus geeignet ist, die Sportschiedsgerichtsbarkeit in ihren Grundfesten zu erschüttern, ${ }^{5}$ entschieden, dass diese Athletenvereinbarungen unwirksam sind, weil sie mit Art. 6 EMRK nicht vereinbar seien.

Angesichts dieser drängenden und kontrovers diskutierten Fragen will der Beitrag eine umfassende Einordnung der Sportschiedsgerichtsbarkeit in den Kontext des Art. 6 EMRK vornehmen, wobei grundsätzlich zwei Problemschwerpunkte in den Blick genommen wer-

1 Vgl. http://www.sueddeutsche.de/sport/sport-kompakt-training-ohne-neuer-1.952797 (17.3.2014).

2 EGMR, Nr. 67474/10, Pechstein/Suisse.

3 Hierzu FAZ v. 29.10.2013, Pechstein-Petition: Explosive Erklärung.

4 LG München I v. 26.2.2014, 37 O 28331/12.

5 Hierzu Adolphsen, Das Aus für die Sportschiedsgerichtsbarkeit?, LTO v. 28.2.2104. 
den sollen. Zum einen stellt sich für die Sportler die Frage, ob der EGMR als zusätzliche Instanz in Sportgerichtsverfahren dienen und überprüfen kann, ob der CAS, weitere internationale Schiedsgerichte oder das Schweizer Bundesgericht im Einklang mit der Konvention Recht gesprochen haben. Diese Frage wird sich auch der EGMR in dem anhängigen Verfahren Pechsteins gegen die Schweiz stellen müssen. Für die Beantwortung rückt dabei insbesondere Art. 6 EMRK in den Vordergrund, wonach jeder Person garantiert wird, dass über Streitigkeiten in Bezug auf ihre zivilrechtlichen Ansprüche und Verpflichtungen oder über eine gegen sie erhobene strafrechtliche Anklage von einem unabhängigen und unparteiischen, auf Gesetz beruhenden Gericht in einem fairen Verfahren, öffentlich und innerhalb angemessener Frist verhandelt werden muss. Von besonderer Bedeutung ist dabei, ob sich das Sportschiedsgericht überhaupt nach den Vorgaben des Art. 6 EMRK zu richten hat (B., C.) und damit Möglichkeiten für Sportler eröffnet werden, Rechtsschutz auf der Grundlage von Art. 6 EMRK nach vorgeschalteten Schiedsurteilen zu ersuchen. Zum anderen will der Beitrag auch den Umfang der Bindung der Sportschiedsgerichte an Art. 6 EMRK skizzieren und dabei insbesondere die derzeit kontrovers diskutieren Athletenvereinbarungen in den Blick nehmen und auf ihre Vereinbarkeit mit der Konvention überprüfen (D.).

\section{B. Das Sportschiedsgericht als Gericht im Sinne des Art. 6 Abs. 1 EMRK ${ }^{6}$}

Unbestritten will die EMRK nicht die Lösung von Konflikten durch die Einsetzung von Schiedsgerichten unter gleichzeitigem Ausschluss staatlicher Justiz verbieten. In diesem Sinne hat auch der EGMR mehrfach festgestellt, dass

,Article $6[E C H R]$ does not preclude the setting up of arbitration tribunals in order to settle disputes between private entities. " 7

Davon zu trennen ist allerdings die Frage einer unmittelbaren Bindung der Schiedsgerichte an Art. 6 Abs. 1 EMRK. Ausgangspunkt der Überlegung bezüglich einer Bindung ist zunächst der Konventionstext. Dieser erwähnt indes die Schiedsgerichtsbarkeit an keiner Stelle. Auch aus den Materialien zur Entstehungsgeschichte der Konvention lassen sich keine Rückschlüsse auf die Frage ziehen, ob und in welchem Umfang die Schiedsgerichte an die EMRK gebunden sind; deren Bedeutung für die EMRK wurde nicht diskutiert. ${ }^{8}$ Verfehlt wäre es deshalb aber, aus der fehlenden Berücksichtigung der Schiedsgerichtsbarkeit im Rahmen der Ausarbeitung des Konventionstextes auf die grundsätzliche

6 Eine Vielzahl nachfolgender Entscheidungen der Konventionsorgane sind unveröffentlicht, aber unter www.echr. coe.int/ECHR/EN/hudoc abrufbar.

7 Vgl. zuletzt EGMR, Nr.773/03, Regent Company/Ukraine, Rdnr. 54; sowie zuvor EGMR, Nr. 9006/80, Lithgow and others/UK, Rdnr. 201. In diesem Sinne schon EKMR, Nr. 1197/61, $X / R F D A$.

8 Vgl. auch Wedam-Lukic, Arbitration and Article 6 of The European Convention On Human Rights, Arbitration 1998, S. 16; Landrove, in: Besson/Hottelier/Werro (Hrsg.), Human Rights at the Center, 2006, S. 74; Jarrosson, L'Arbitrage et La Convention Européenne des Droits De L'Homme, Rev. arb. 1989, S. 574; Knoepfler, Les Droits de l'homme et l'arbitrage, RSDIE 2007, S. 466; Haas, Internationale Sportschiedsgerichtsbarkeit und EMRK, SchiedsVZ 2009, S. 74. 
Bedeutungslosigkeit der EMRK für die Schiedsgerichtsbarkeit zu schließen. ${ }^{9}$ Schließlich betont der EGMR in ständiger Rechtsprechung, dass die Konvention ein ,living instrument" ist und die dort niedergelegten Rechte und Freiheiten nicht nur ,theoretical or illusory but practical and effective" gewährleistet werden sollen. ${ }^{10}$ Maßgeblich ist deshalb zunächst, ob ein Schiedsgericht tatsächlich ein Gericht im Sinne des Art. 6 Abs. 1 EMRK darstellt.

Artikel 6 Abs. 1 EMRK gewährleistet jeder Person das Recht, dass über Streitigkeiten in Bezug auf ihre zivilrechtlichen Ansprüche und Verpflichtungen oder über eine gegen sie erhobene strafrechtliche Anklage von einem unabhängigen und unparteiischen, auf Gesetz beruhenden Gericht in einem fairen Verfahren, öffentlich und innerhalb angemessener Frist verhandelt wird. Voraussetzung für die Bindung an Art. 6 Abs. 1 EMRK ist deshalb zuvörderst die Eigenschaft eines Schiedsgerichts als „unabhängiges und unparteiisches, auf Gesetz beruhendes Gericht" im Sinne des Art. 6 Abs. 1 EMRK. Für die Einreihung der Schiedsgerichtsbarkeit in den Gerichtsbegriff des Art. 6 Abs. 1 EMRK ist dabei zunächst bedeutsam, dass es weder auf die Bezeichnung des Spruchkörpers, noch auf dessen rechtliche Einordung im innerstaatlichen Recht ankommt. ${ }^{11}$

Entscheidend für die Einordnung der Sportschiedsgerichtsbarkeit in den Kontext des Art. 6 Abs. 1 EMRK ist das Merkmal ,,auf Gesetz beruhendem Gericht“" ${ }^{12}$ Dabei ist weniger die Frage, ob ein Schiedsgericht ein Gericht im Sinne des Art. 6 Abs. 1 EMRK darstellt, ${ }^{13}$ problematisch, sondern die Voraussetzung ,established by law“. Denn angesichts der Tatsache, dass privat eingesetzte (Sport-)Schiedsgerichte nicht aufgrund gesetzlicher Vorschriften konstituiert werden, sondern letztlich auf dem Willen der Parteien beruhen, wird von einigen Stimmen in der Literatur die Eigenschaft von echten privaten Schiedsgerichten als Gericht „established by law“ im Sinne des Art. 6 Abs. 1 EMRK abgelehnt. ${ }^{14}$ Konsequenterweise versteht diese Auffassung gesetzlich angeordnete Schiedsgerichte hingegen als auf gesetzlichen Vorschriften beruhende Gerichte gemäß Art. 6

9 Andeutend Distler, Private Schiedsgerichtsbarkeit und Verfassung, 2000, S. 265.

10 Vgl. EGMR, Nr. 6694/74, Artico/Italy, Rdnr. 33; EGMR, Nr. 59320/00, Von Hannover/Germany $=$ IIC 2004, 672, Rdnr. 36.

11 Vgl. Grabenwarter/Pabel, Europäische Menschenrechtskonvention, 5. Aufl. 2012, § 24, Rdnr. 27.

12 In der englischen Originalfassung, die im Gegensatz zur deutschen Fassung verbindlich ist (vgl. Art. 59 Abs. 4 EMRK), lautet die relevante Passage: ,established by law“. Vor diesem Hintergrund wird hiernach die englische Formulierung verwendet.

13 Dies hat der EGMR mit Blick auf gesetzlich angeordnete Schiedsgerichte mehrfach festgestellt; vgl. etwa EGMR, Nr. 9006/80, Lithgow and others/UK, Rdnr. 201: „Again, [the Court] recalls that the word ,tribunal' in Article 6 para. 1 is not necessarily to be understood as signifying a court of law of the classic kind [...]; thus, [national law] may compromise a body to set up a limited number of specific issues." Siehe ferner EGMR, Nr. 773/03, Regent Company/Ukraine, Rdnr. 54.

14 In diesem Sinne Jarrosson, (Fn. 8), S. 592, Rdnr. 35; Jacot-Guillarmod, in: Matscher/Petzold (Hrsg.), Mélanges Wiarda, 1988, S. 290 f.; Landrove, (Fn. 8), S. 79 f.; Grothe/Frohn, Causa Sport 2008, S. 107, Fn. 39; Robinson/Kasolowsky, Will the United Kingdom's Human Rights Act Further Protect Parties to Arbitration Proceedings, Arbitration International 2002, S. 456 f.; Distler, (Fn. 9), S. 266; Tochtermann, Die Unabhängigkeit und Unparteilichkeit des Mediators, 2008, S. 38 f. Unklar Reiner, Schiedsverfahren und rechtliches Gehör, ZfRV 2003, S. 60, der wohl schon davon ausgeht, dass Schiedsgerichte keine „Gerichte“ i.S.d. Art. 6 EMRK sind. 
Abs. 1 EMRK. Auf den ersten Blick mag diese Differenzierung einleuchten, zumindest dann, wenn die Formulierung ,established by law" strikt im wörtlichen Sinne verstanden wird. Zweifelhaft ist aber schon, ob diese Formulierung tatsächlich darauf abzielt, auf privaten Willen zurückzuführende Schiedsgerichte dem Anwendungsbereich des Art. 6 Abs. 1 EMRK zu entziehen. Hintergrund dieser Anforderung war nämlich die Intention der Gründungsstaaten, die Errichtung von, ,ad-hoc“-Gerichten durch exekutive Organe, die für einen spezifischen Fall nachträglich konstituiert werden, zu verhindern. ${ }^{15}$ Zweck der gesetzlichen Vorausregelung ist demnach das Verbot von Ausnahmegerichten und weniger der Ausschluss der Schiedsgerichtsbarkeit vom Gerichtsbegriff nach Art. 6 Abs. 1 der Konvention.

Daneben zeichnet den Sport in vielen Bereichen die Verflechtung zwischen staatlicher und privater Zuständigkeit aus. ${ }^{16}$ Angesichts der vielschichtigen Verzahnungen erweist sich die Differenzierung zwischen unmittelbar und lediglich mittelbar auf gesetzlichen Vorschriften beruhenden Schiedsgerichten als ungeeignet, denn die Übergänge zwischen diesen beiden Formen sind letztlich fließend. ${ }^{17}$ Dies gilt im Übrigen nicht nur für die Materie Sport; auch der rechtsvergleichende Blick bezüglich der Unterscheidung staatlich festgesetzter und privatautonom begründeter Schiedsgerichtsbarkeit im Anwendungsbereich anderer Abkommen ${ }^{18}$ zeigt, dass dort ebenfalls keine trennscharfe Abgrenzung im Hinblick auf die schiedsgerichtliche Begründungsweise erfolgt. ${ }^{19}$

Eng verknüpft mit der Verzahnung staatlicher und sportinterner Konfliktlösungsmechanismen ist die Begründung der Schiedszuständigkeit für den betroffenen Sportler selbst. Häufig wird - wie bereits eingangs erwähnt - die Anmeldung zur Teilnahme an sportlichen (Groß-)Veranstaltungen mit der Zustimmung zur schiedsgerichtlichen Streitbeilegung im Konfliktfall verbunden. ${ }^{20}$ Dem Sportler bleibt in der Regel damit keine andere Wahl, als

15 Im Gegensatz zu vergleichbaren Abkommen - etwa Art. 8 AMRK: ,previously established by law" - formuliert dies Art. 6 EMRK nicht eindeutig. Vgl. aber EKMR, Nr. 7360/76, Zand/Österreich, DR 15, S. 70, Rdnr. 69: „It is the object and purpose of the clause in Art. 6(1) requiring that the courts shall be established by law' that the judicial organization in a democratic society must not depend on the discretion of the Executive." Zum Verbot von Ausnahmegerichten siehe auch EGMR, Nr. 9006/80, Lithgow and others/UK, Rdnr. 201. Vgl. hierzu ferner Müßig, Gesetzlicher Richter Ohne Rechtsstaat?, 2007, S. 37 ff. m.w.N.

16 Im Einzelnen Nolte, Staatliche Verantwortung im Bereich Sport, 2004, passim.

17 Zutreffend Haas, (Fn. 8), S. 75. Ähnlich Besson, Arbitration and Human Rights, ASA Bulletin 2006, S. 398. Vgl. etwa den UNESCO-Konventionsentwurf der Generalversammlung zur Bekämpfung des Dopings im Sport - UNESCO-Res., Records of the General Conference 2004, Vol. 1, Rdnr. 9 - in welchem sich die Staaten verpflichten, das von der WADA geschaffene AntiDoping-Regelwerk (World Anti-Doping Code) anzuerkennen; dieses setzt wiederum den CAS als Letztentscheidungsinstanz für Streitigkeiten ein.

18 Vgl. etwa das New Yorker Übereinkommen über die Anerkennung und Vollstreckung ausländischer Schiedssprüche v. 10.6.1958, BGB1. II 1961, 122; in Kraft seit dem 28.9.1961.

19 Ausführlich und insbesondere auf das UNÜ-Abkommen und die Handelsschiedsgerichte der ehemaligen Ostblockstaaten eingehend siehe Haas, (Fn. 8), S. 75 f.; Samuel, Arbitration, Alternative Dispute Resolution Generally and the European Convention on Human Rights - An AngloCentric View, Journal of International Arbitration 2004, S. 424.

20 Vgl. etwa die „Entry-Form“ für die Teilnahme an Olympischen Spielen, die für wettbewerbsrelevante Streitigkeiten die Zuständigkeit des CAS anordnet; vgl. hierzu nur Haas, Zur Einführung von Schiedsklauseln durch Satzungsänderungen in Vereinen, ZGR 2001, S. 336 f. Zur konventionsrechtlichen Zulässigkeit solcher Schiedsklauseln siehe die Ausführungen unter D. 
der Unterwerfung unter die Sportschiedsgerichtsbarkeit zuzustimmen, will er an dem Wettkampf tatsächlich partizipieren. Von daher nähert sich diese Form der „Schiedsvereinbarung“ im internationalen Sportrecht in funktioneller Hinsicht der staatlich angeordneten Schiedsgerichtsbarkeit an. Vor diesem Hintergrund ist es aus der Sicht der EMRK letzten Endes unerheblich, ob das zur Entscheidung berufene Schiedsgericht aufgrund zwingender gesetzlicher Vorgaben konstituiert worden ist oder aber durch die faktisch erzwungene Zustimmung auf ,privatautonomen“ Wege. ${ }^{21}$

Ausschlaggebend für die Einordnung von Schiedsgerichten als ein Gericht „established by law" im Sinne des Art. 6 Abs. 1 EMRK ist letztlich die Tatsache, dass sich die Zuweisung zwar auf eine von den Parteien geschlossene Übereinkunft zurückführen lässt, gleichwohl die Schiedsgerichte aber nicht außerhalb der staatlichen Rechtsordnung agieren. Vielmehr fußt schon die generelle Zulässigkeit schiedsgerichtlicher Entscheidungsfindung bei gleichzeitig eingeschränkter staatlicher Überprüfungskompetenz in der verfassungsrechtlich zugestandenen Vertragsfreiheit. Die Anforderungen an die Besetzung und das einzuhaltende Verfahren sind ebenfalls gesetzlichen Vorgaben zu entnehmen. Auch die Schiedssprüche selbst und die daran anschließenden Anerkennungs- und Vollstreckungsmöglichkeiten im innerstaatlichen Rechtsraum knüpfen an diese Garantie an, sind aber zugleich auch im Lichte der Vorgaben des staatlichen Ordnungsrahmens zu sehen. Mithin zeigt sich, dass sich die Idee der Schiedsgerichtsbarkeit zumindest in indirekter Weise auf gesetzliche Normen zurückführen lässt ${ }^{22}$ und damit das Sportschiedsgericht sehr wohl ein „tribunal established by law" darstellt.

\section{Bindung der Schiedsgerichte an Art. 6 Abs. 1 EMRK}

Unterfallen demnach Schiedsgerichte nach zutreffender Ansicht dem Gerichtsbegriff des Art. 6 Abs. 1 EMRK, ${ }^{23}$ so bedeutet dies gleichfalls nicht, dass die Garantien des Art. 6 Abs. 1 der Konvention nun zugleich auch für die Schiedsgerichtsbarkeit im Ganzen gelten. Unbestritten ist Art. 6 Abs. 1 EMRK in denjenigen Konstellationen von Bedeutung, die staatlich angeordnete Schiedsgerichte zum Gegenstand haben, selbst dann, wenn die Parteien die Möglichkeit hatten, die Schiedsrichter selbst zu bestimmen. In diesen Fällen sind die durch staatlichen Befehl zur Entscheidung berufenen Schiedsgerichte an Art. 6 Abs. 1 EMRK gebunden, womit sich das Gerichtsverfahren an den Anforderungen des Art. 6

21 Treffend formuliert dies Besson, (Fn. 17), S. 398: „[Sport-related Arbitration is] not, compulsory “ but ,forced ' arbitration." Diese funktionale Betrachtungsweise befürworten auch Haas, (Fn. 8), S. 76; Rigozzi, L'arbitrage international en matière du sport, 2005, S. 473; Knoepfler, (Fn. 8), S. $465 \mathrm{f}$.

22 Cambi Favre-Bulle, in: Cambi Favre-Bulle et al. (Hrsg.), L'arbitrage et La Convention européenne des droits de l'homme, 2001, S. 73.

23 Ebenso Haas, (Fn. 8), S. 75 f.; Lambert, in: Cambi Favre-Bulle et al., (Fn. 22), S. 17 f.; Knoepfler, (Fn. 8), S. 467 f.; Cambi Favre-Bulle, (Fn. 22), S. 73; Besson, (Fn. 17), S. 401; Jaksic, Arbitration and Human Rights, 2002, S. 185 ff.; Ambrose, Arbitration and the Human Rights Act, L.M.C.L.Q. 2000, S. 473 ff.; Poncet/Cambi Favre-Bulle, in: De Fontbressin et al. (Hrsg.), Mélanges Lambert, 2000, S. 668; Rigozzi, (Fn. 21), S. 472 f. Wohl auch Habscheid, in: Gerhardt et al. (Hrsg.), Festschrift Henckel, 1995, S. 348 f. 
Abs. 1 der Konvention messen lassen muss. ${ }^{24}$ Anknüpfungspunkt hierfür ist Art. 1 EMRK, wonach die Vertragsparteien allen ihrer Hoheitsgewalt unterstehenden Personen die in der Konvention bestimmten Rechte und Freiheiten zusichern. Verlagern staatliche Vorschriften Rechtsprechungsgewalt in den schiedsgerichtlichen Bereich, so lässt dies ihre Verpflichtung aus der Konvention nicht entfallen. ${ }^{25}$ Vielmehr muss sodann auch im schiedsgerichtlichen Verfahren sichergestellt werden, dass die grundlegenden Verfahrensgarantien des Art. 6 Abs. 1 EMRK gewährleistet werden. ${ }^{26}$

Umstrittener und weitaus komplexer ist die Frage, ob sich die Garantien des Art. 6 Abs. 1 EMRK auch auf die ,echte“ freiwillige Schiedsgerichtsbarkeit beziehen. Voraussetzung dafür ist, dass diese Schiedsstellen ebenfalls Adressaten des Art. 6 Abs. 1 der Konvention sind und folglich an die EMRK gebunden sind. Vor diesem Hintergrund wird zunächst die Rechtsprechung nationaler Gerichte sowie die Auffassung der Rechtsprechungsorgane der EMRK beleuchtet, um sodann den Stand der rechtswissenschaftlichen Diskussion darzustellen. Auf diesen Prämissen aufbauend wird schließlich eine eigene Bewertung dargelegt.

\section{Nationale Gerichtsentscheidungen}

Um zu bestimmen, ob ,private“ Schiedsgerichte Adressaten von Art. 6 EMRK sind, lohnt sich ein (rechtsvergleichender) Blick in die jeweilige nationale Spruchpraxis. Denn im Rahmen der Auslegung der Konventionsrechte sind nach Auffassung des EGMR auch „gemeineuropäische Rechtsauffassungen“ zu berücksichtigen. ${ }^{27}$ Soweit ersichtlich, hat sich jedoch zumindest der BGH in denjenigen Fällen, in denen die Richter Schiedssprüche aufgrund eines unzulässigen Verfahrens ( $\$ 1059$ Abs. 2 Nr. 1 ZPO) beanstandet haben, nicht (auch) auf Art. 6 Abs. 1 EMRK berufen, selbst wenn dort entsprechende Vorgaben postuliert werden.

Von grundlegender Bedeutung sind die Entscheidungen des Schweizer Bundesgerichts, da dieses die Revisionsinstanz für die Entscheidungen des CAS ist und sich somit naturgemäß häufig mit der Frage der Bindung der Sportschiedsgerichte an die EMRK ausein-

24 Vgl. EKMR, Nrn. 8588/79 und 8589/79, Bramelid and Malmström/Sweden; EGMR, Nr. 9006/80, Lithgow and others/UK, Rdnrn. 192, 200 ff.; EGMR, Nr. 33745/96, Scarth/UK, Rdnr. 29; EGMR, Nr. 773/03, Regent Company/Ukraine, Rdnr. 54 ff.

25 Die Verantwortlichkeit der Konventionsstaaten hat der EGMR mit Blick auf andere funktionale Hoheitsträger anerkannt; vgl. etwa EGMR, Nr. 13134/87, Castello-Roberts.

26 Ähnlich verhält es sich bei der Verantwortlichkeit der Konventionsstaaten mit Blick auf die EU, die bis jetzt noch selbst nicht Vertragspartei der EMRK ist. Auch hier darf die Übertragung von Kompetenzen nicht dazu führen, dass die Rechte der EMRK nicht mehr gewährleistet werden können; die Verantwortung der Mitgliedstaaten für die konventionskonforme Ausübung der übertragenden Hoheitsbefugnisse besteht vielmehr fort, vgl. zuletzt EGMR, Nr. 45036/98, Bosphorus/Ireland, Rdnr. 154. Zur Rechtsprechung des EGMR mit Blick auf das EU-Recht vgl. ferner Schmahl, Grundrechtschutz im Dreieck von EU, EMRK und nationalem Verfassungsrecht, EuR-Beiheft 1/2008, S. 26 ff.

27 EGMR, Nr. 22985/93, Sheffield and Horsham/UK, Rdnr. 57 f. Siehe auch Kleine-Kosack, Verfassungsbeschwerden und Menschenrechtsbeschwerden, 2007, Rdnr. 1392. 
andersetzen muss. ${ }^{28}$ Zunächst verneinte das Bundesgericht noch die Anwendbarkeit des Art. 6 Abs. 1 EMRK im Hinblick auf die Schiedsgerichtsbarkeit. ${ }^{29}$ Diese Sichtweise bestätigte das Gericht in einem Urteil aus dem Jahre 1986. ${ }^{30}$ Dort stellte das Bundesgericht explizit fest, dass Schiedsgerichte nicht Adressaten der EMRK seien und folglich eine Berufung auf Art. 6 Abs. 1 EMRK ausscheide. ${ }^{31}$

Fünf Jahre später bot sich dem Bundesgericht erneut die Möglichkeit, zum Verhältnis zwischen EMRK und Schiedsgerichtsbarkeit Stellung zu nehmen. Gegenstand des Verfahrens war ein Teilschiedsspruch eines der Verfahrensordnung der Internationalen Handelskammer in Paris unterstehenden Schiedsgerichts mit Sitz in Zürich. ${ }^{32}$ Die Beschwerdeführerin beklagte die Befangenheit der Schiedsrichter und rügte neben der Verletzung nationalen Rechts auch die Verletzung von Art. 6 Abs. 1 EMRK. In seinem Urteil entschied das Bundesgericht - ohne auch nur mit einem einzigen Wort auf die vorangegangenen Entscheidungen einzugehen - dass sich

„die aus Art. 6 EMRK fließenden Garantien nicht blo[ß] auf staatliche Gerichte beziehen, sondern auch auf private Schiedsgerichte, $[\ldots][$ da] diese dieselbe Gewähr für eine unabhängige Rechtsprechung bieten müssen“. ${ }^{33}$

Deswegen befand das Bundesgericht die Klage für erfolgreich, da das Schiedsgericht nicht ordnungsgemäß besetzt gewesen sei; Art. 6 Abs. 1 EMRK beinhalte aber auch den fundamentalen Anspruch auf vorschriftsgemäße Zusammensetzung der Spruchkammer. ${ }^{34}$

Diese Rechtsauffassung bestätigte das Schweizer Bundesgericht indirekt in einer weiteren staatsrechtlichen Beschwerde aus dem Jahre 2000. Dort beantragte die Beschwerdeführerin die Aufhebung des Schiedsspruchs eines nach den Regeln der Internationalen Handelskammer (ICC) zur Entscheidung berufenen Schiedsgerichts mit Sitz in Zürich, da sie die Unabhängigkeit eines vom Schiedsgericht eingesetzten Sachverständigen anzweifelte. ${ }^{35}$ In seiner Entscheidung wiederholte das Bundesgericht zwar nicht ausdrücklich, dass Schiedsgerichte an Art. 6 Abs. 1 EMRK gebunden sind, aber die Richter stellten fest, dass

„nach der Rechtsprechung des Bundesgerichts die Verfahrensgarantie [...] von Art. 6 Ziff. 1 EMRK sinngemä[[$[\beta]$ auch auf das Erfordernis der Unabhängigkeit und Unparteilichkeit von Sachverständigen angewendet wird. “36

Bezieht sich das Bundesgericht folglich aber auf Anforderungen an die Unabhängigkeit und Unparteilichkeit von Gutachtern, die sich aus Art. 6 Abs. 1 der EMRK ergeben solle,

28 Dies gilt im Übrigen nicht nur für den Sport. Auch in anderen Bereichen ist die Schweiz bevorzugter Ort für die Ansiedelung von Schiedsgerichten.

29 BG v. 9.2.1984 (unveröffentlicht); Bezug genommen in BGE 112 Ia, S. 168.

30 BGE 112 Ia, S. $166 \mathrm{ff}$.

31 Ibid., S. 168: „[La Convention] ne saurait donc s'appliquer à un tribunal arbitral dont les membres sont désignés librement par les parties". Genau genommen versteht damit das BG Schiedsgerichte schon nicht als ein ,auf Gesetz beruhendes Gericht“i.S.d. Art. 6 Abs. 1 EMRK.

32 Vgl. BGE 117 Ia, S. 166 ff.

33 Ibid., S. 168.

34 Ibid., S. 170.

35 BGE 126 III, S. $249 \mathrm{ff}$.

36 Ibid., S. 253. 
so kann dies zu der Überlegung führen, dass auch die Schiedsgerichte Adressaten von Art. 6 Abs. 1 EMRK sind.

Deutlicher wurde das Bundesgericht in einer weiteren Entscheidung aus dem Jahre 2004. ${ }^{37}$ Dort rügte die Beschwerdeführerin, dass das Schiedsgericht wegen Befangenheit des Obmanns ordnungswidrig zusammengesetzt gewesen sei. Das Bundesgericht stellt in diesem Zusammenhang fest, dass

„[Art. 6 Abs. 1 EMRK] nicht nur für staatliche Gerichte [gilt], sondern auch für private Schiedsgerichte, deren Entscheide jenen der staatlichen Rechtspflege hinsichtlich Rechtskraft und Vollstreckbarkeit gleichstehen und die deshalb grundsätzlich dieselbe Gewähr für eine unabhängige Rechtsprechung bieten müssen“. ${ }^{38}$

Urteile aus jüngster Zeit erwecken indes zunehmend den Eindruck, als ob das Bundesgericht wieder zu seiner Ausgangsentscheidung aus dem Jahre 1986 zurückgekehrt ist. In der Rechtssache Abel Xavier rügte dieser einen Schiedsspruch des CAS, nachdem das Gericht eine durch die UEFA verhängte Dopingsperre dem Grunde nach bestätigt hatte. Der Beschwerdeführer berief sich auf eine Verletzung des ordre public und beantragte, die CASEntscheidung für nichtig zu befinden. Diesmal stellte das Bundesgericht explizit fest, dass

„l'art. 6 par. 1 CEDH ne se rapporte pas à la procédure devant un tribunal arbitral““.39

Diese fehlende unmittelbare Bindung der Schiedsgerichte an Art. 6 Abs. 1 EMRK bestätigte das Bundesgericht in einer Entscheidung aus dem Jahre 2006. Dort beantragte der Beschwerdeführer, ein Langstreckenreiter aus den Vereinigten Arabischen Emiraten, einen zuvor vom CAS ergangenen Schiedsspruch für nichtig zu erklären, da der CAS weder zuständig noch ordnungsgemäß besetzt gewesen sei. Daneben rügte er eine Verletzung der Begründungspflicht des CAS und damit die Verletzung seines Anspruchs auf rechtliches Gehör, womit jedenfalls auch Art. 6 Abs. 1 EMRK betroffen sei ${ }^{40}$ DasBundesgerichtentschied, dass mit der staatsrechtlichen Beschwerde nicht direkt eine Verletzung der EMRK gerügt werden könne, denn die Bestimmung des Art. 6 Abs. 1 EMRK sei nicht auf das Verfahren der freiwilligen Schiedsgerichtsbarkeit anwendbar. ${ }^{41}$ Auch in der Causa Pechstein, die insbesondere in der deutschen Öffentlichkeit aufmerksam verfolgt wurde, nahm das Bundesgericht zum Verhältnis von Art. 6 Abs. 1 EMRK und der Schiedsgerichtsbarkeit Stellung. Bevor das Gericht das nähere Vorbringen Pechsteins würdigte, stellte es fest, dass mit einerBeschwerde gegen einen internationalen Schiedsentscheid nicht direkteine Verletzung derEMRK gerügt werden könne, insbesondere auch nicht des Art. 6 Abs. 1 EMRK. ${ }^{42}$

37 BG v. 14.12.2004, 4P.208/2004//ma (www.bger.ch).

38 Ibid., Rdnr. 4.1.

39 BG v. 11.6.2001, 4P.64/2001, Rdnr. 2.

40 BG v. 9.3.2006, 4P.105/2006 = Causa Sport 2006, 575, Rdnr. 7.3.

41 Ibid.

42 BG v. 10.2.2010, 4A_612/2009, Rdnrn. 2.4.1, 4.1. Bestätigt in Entscheidung des BG v. 2.6.2010, 4A_320/2009, Rdnrn. 1.5.3,2: ,Die Beschwerdeführer rügen zu Unrecht unter Berufung auf Art. 6 EMRK eine Verletzung des Anspruchs auf ein öffentliches Verfahren, da diese Bestimmung nach zutreffendem Verständnis der bundesgerichtlichen Rechtsprechung auf Verfahren der freiwilligen Schiedsgerichtsbarkeit nicht anwendbar ist.“Ebenso BG v. 19.7.2010, 4A_43/2010, Rdnr. 3.6.1.; BG v. 19.4.2011 4A_404/2010, Rdnr. 3.5.3. 
Wenngleich also das Bundesgericht zunächst keine einheitliche Linie bezüglich des Verhältnisses von Schiedsgerichtsbarkeit und EMRK verfolgte, muss angesichts der jüngeren Rechtsprechung davon ausgegangen werden, dass das Gericht eine unmittelbare Bindung an Art. 6 Abs. 1 EMRK ablehnt. ${ }^{43}$

Auch französische Gerichte verneinen eine unmittelbare Bindung an Art. 6 Abs. 1 EMRK. ${ }^{44}$ So monierte etwa ein amerikanischer Beschwerdeführer in einem iranischamerikanischen Streitfall vor französischen Zivilgerichten das vor einem nicht-obligatorischen Schiedsgericht verhandelte Verfahren widerspreche dem in Art. 6 Abs. 1 EMRK niedergelegten Gebot des zügigen Verfahrens. ${ }^{45}$ Der Cour d'appel de Paris hielt die ablehnende Entscheidung der Eingangsinstanz (Tribunal de grande instance de Paris) ${ }^{46}$ aufrecht; in diesem Zusammenhang stellte das Gericht ausdrücklich fest, dass die Garantien aus Art. 6 Abs. 1 EMRK lediglich Staaten verpflichten, nicht aber private Dritte, worunter auch die Schiedsgerichtsbarkeit falle. ${ }^{47}$ Auch das Berufungsgericht (Cour de Cassation) hat die Entscheidung des Cour d'appel de Paris bestätigt und eine unmittelbare Bindung des Schiedsgerichts an Art. 6 EMRK abgelehnt. ${ }^{48}$

In einem Verfahren vor dem österreichischen Obersten Gerichtshof (OGH) berief sich ein Beschwerdeführer ebenfalls auf Art. 6 Abs. 1 EMRK. Vorangegangen war ein schiedsgerichtliches Verfahren, dessen Gegenstand die rechtliche Zuordnung des Eigentums an einem Gemälde Gustav Klimts war. Gegen den nachteiligen Schiedsspruch des eingesetzten Schiedsgerichts machte die unterlegene Partei vor dem OGH geltend, dass weder die Schiedsvereinbarung noch das Schiedsverfahren den Verfahrensgarantien des Art. 6 Abs. 1 EMRK entspreche. ${ }^{49}$ Wiewohl der OGH in den Urteilsgründen zwar nicht explizit die Bindung der Schiedsgerichte an Art. 6 Abs. 1 EMRK verneint, lässt die Entscheidung Rückschlüsse auf die Haltung des OGH in dieser Frage zu. Die vom Beschwerdeführer angeführte Entscheidung des EGMR, die das Begehren desselben und die Bindung an Art. 6 Abs. 1 EMRK unterstützen sollte, war nämlich für den OGH nicht maßgeblich:

„Soweit sich die außerordentliche Revision auf die Entscheidung EuGRZ 1983, 428 Nr 91 (Bramelid und Malmström/S) hinsichtlich der Konventionswidrigkeit der Schiedsrichterbestellung im vorliegenden Fall bezieht, ist ihr entgegen zu halten, dass die zitierte Entscheidung nicht nur einen Fall sogenannter Zwangsschiedsgerichtsbarkeit zum Gegenstand hatte, sondern

43 Die Entscheidung des BG v. 20.6.2008,9C_132/2008, ist kein Beleg für die Anwendbarkeit des Art. 6 Abs. 1 EMRK, denn dort entschied das BG über die Rechtmäßigkeit des Verfahrens vor einem wegen Art. 89 Abs. 4 KGV staatlich angeordneten Schiedsgericht. In diesen Fällen gilt Art. 6 Abs. 1 EMRK ohnehin.

44 In Tribunal de grande instance de Paris v. 20.10.1997, Boulois/UNESCO = Rev. arb. 1997, 575, Rdnr. 9 (mit Anmerkung Jarrosson) entschied das Tribunal de grande instance de Paris, dass der staatliche Richter die Garantien des Art. 6 Abs. 1 EMRK mit Blick auf schiedsgerichtliche Verfahren zu respektieren habe.

45 Näher hierzu Briner/Schlabrendorff, in: Briner et al. (Hrsg.), Liber Amicorum für Böckstiegel, 2001, S. 90; Haas, (Fn. 8), S. 76; Samuel, (Fn. 19), S. 424.

46 Tribunal de grande instance de Paris v. 21.5.1997 = Rev. arb. 1997, $417 \mathrm{ff}$.

47 Cour d'appel de Paris v. 15.9.1998 = Rev. arb. 1999, 113, Rdnr. 27: „La CEDH s'impose aux Etats signataires et non pas à une association qui ne constitue pas une juridiction."

48 Cour de Cassation v. 20.2.2001 = Gaz Pal 2001, 119 ff.: Art. 6 der Konvention „ne concerne que les Etats et les juridictions étatiques."

49 Vgl. OGH v. 1.4.2008-5Ob272/07x. 
dort für die Schiedsbeklagte von einer staatlichen Stelle ein Schiedsrichter bestellt wurde, während die Schiedsklägerin, ein führendes schwedisches Wirtschaftsunternehmen, eine Person ihres Vertrauens zu ihrem Schiedsrichter bestellen konnte. Der Fall ist dem gegenständlichen also keineswegs vergleichbar.“

Die mangelnde Vergleichbarkeit der Entscheidung Bramelid and Malmström/Sweden, auf welche der OGH hinweist, bezieht sich zwar nicht direkt auf die Frage der Bindung an die EMRK, sondern vermutlich auf die fehlende Vergleichbarkeit der beiden Fälle im Hinblick auf die Schiedsrichterbestellung. Aus der Formulierung „dass die zitierte Entscheidung nicht nur einen Fall sogenannter Zwangsschiedsgerichtsbarkeit zum Gegenstand hatte“ lässt sich aber auch schließen, dass der OGH wohl davon ausgeht, dass nur staatlich angeordnete Schiedsgerichte unmittelbar von Art. 6 Abs. 1 EMRK erfasst werden und damit gerade nicht diejenigen Schiedsstellen, die auf privatautonomer Basis konstituiert worden sind.

\section{Rechtsauffassung der Rechtsprechungsorgane der EMRK}

Im Ergebnis lässt sich festhalten, dass staatliche Gerichte bisher dazu neigen, eine unmittelbare Bindung der Schiedsgerichte an Art. 6 Abs. 1 EMRK abzulehnen. Allerdings kann aufgrund der beschränkten Anzahl an Entscheidungen wohl kaum von einer gemeineuropäischen Rechtsauffassung bezüglich der Einordung der Schiedsgerichtsbarkeit in den Anwendungsbereich von Art. 6 Abs. 1 EMRK gesprochen werden. Die Judikate nationaler Gerichte können darüber hinaus ohnehin nur als Indiz für die Frage einer Bindung von Schiedsgerichten an Art. 6 Abs. 1 EMRK dienen. Denn die Auslegung der EMRK und der darin enthaltenden Begriffe ist den Rechtsprechungsorganen der EMRK vorbehalten. Die Bestimmung der Adressatenstellung im Rahmen des Art. 6 Abs. 1 EMRK erfolgt also auf konventionsautonome Weise.

Im Jahre 1962 war die Europäische Kommission für Menschenrechte (EKMR) in $X / R F D A$ erstmalig dazu aufgerufen, schiedsgerichtliche Entscheidungen in den Kontext der Konvention einzuordnen. ${ }^{50}$ Gegenstand des Verfahrens war die Beschwerde eines deutschen Lehrers, der in Spanien an einer deutschen Schule beschäftigt war. Der Anstellungsvertrag enthielt eine Schiedsklausel, wonach die Zuständigkeit bei Streitfällen einem Vertreter der Bundesrepublik Deutschland übertragen wurde. Nachdem es zum Streitfall kam, rief der Lehrer - nach erfolglosem schiedsgerichtlichen Verfahren - ein spanisches Arbeitsgericht an, das die Schiedsklausel zwar als nichtig ansah, sich aber letztlich aus anderen Gründen für unzuständig erklärte. Auch vor deutschen Gerichten fand der Lehrer kein Gehör, insbesondere wurde die Entscheidung des spanischen Gerichts im Hinblick auf die Nichtigkeit der Schiedsklausel nicht anerkannt. Der Beschwerdeführer rügte anschließend die missbräuchliche Entziehung des spanischen Richters durch die deutschen Behörden. In der Entscheidung brachte die Kommission zunächst zum Ausdruck, dass es aus Sicht der Konvention grundsätzlich nicht zu beanstanden sei, dass private Streitigkeiten unter Ausschluss staatlicher Gerichtsbarkeit in privatautonomer Weise geregelt wer-

50 EKMR, Nr. 1197/61, X/RFDA, D.R. 8, S. 68 ff. Zur Entscheidung vgl. auch Matscher, in: Habscheid/Schwab (Hrsg.), Festschrift Nagel, 1987, S. 238 f. 
den. ${ }^{51}$ Auf die Frage der unmittelbaren Bindung von privaten, echten Schiedsgerichten an Art. 6 Abs. 1 EMRK ging die Kommission jedoch ebenso wenig näher ein wie auf eine mögliche mittelbare Drittwirkung des Art. 6 Abs. 1 EMRK; vielmehr untersuchte sie ausführlich die Möglichkeit des Verzichts von Verfahrensrechten. ${ }^{52}$

In der Rechtssache Bramelid and Malmström/Sweden ${ }^{53}$ beruhte die schiedsgerichtliche Zuständigkeit zwar auf gesetzlicher Anordnung. Gerade diese Tatsache nahm die Kommission aber zum Anlass, die Unterschiedlichkeit zwischen staatlich angeordneter und freiwilliger, echter Schiedsgerichtsbarkeit vor dem Hintergrund des Art. 6 Abs. 1 EMRK zu betonen:
„Furthermore, the Commission notes that a distinction must be drawn between voluntary ar- bitration and compulsory arbitration. Normally Article 6 poses no problem where arbitration is entered into voluntarily. If, on the other hand, arbitration is compulsory in the sense of being required by law, as in this case, the parties have no option but to refer their dispute to an Arbitration Board, and the Board must offer the guarantees of a legal obligation. "54

Die Kommission bringt darin zum Ausdruck, dass nur die gesetzlich angeordneten Schiedsgerichte die in Art. 6 Abs. 1 EMRK niedergelegten Rechte garantieren müssen; die echte, freiwillige Schiedsgerichtsbarkeit wirft im Lichte des Art. 6 Abs. 1 EMRK offensichtlich keine Probleme auf.

In der Entscheidung $R /$ Switzerland ${ }^{55}$ bestätigte die Kommission den Verzicht auf eine unmittelbare Überprüfungskompetenz privater Schiedssprüche. Gegenstand des Verfahrens war eine Schiedsvereinbarung, die das Schiedsgericht dazu ermächtigte, den Preis für den Kauf von Gesellschaftsanteilen zu bestimmen sowie den näheren Verkaufsablauf festzulegen. Das Verfahren zog sich - auch wegen der Einbeziehung staatlicher Gerichtsbarkeit - über einen beträchtlichen Zeitraum hin, was der Beschwerdeführer zum Anlass nahm, die überlange Verfahrensdauer bis zur schiedsgerichtlichen Entscheidungsfindung zu rügen. Die Kommission stellte fest,
„that the arbitration proceedings do not fall within the scope of Article 6 para. 1, since the parties, of their own free will, validly entrusted the arbitrators the determination of their civil rights and obligations. The Commission nevertheless considers that [...] account must taken also $[\ldots]$ of the legislative framework for such proceedings. ${ }^{\text {". } 56}$

Indem die Kommission nur die Verantwortlichkeit des Konventionsstaates für die staatlichen Gerichte thematisiert, bringt sie - auch weil sie erneut die Unterschiedlichkeit zwischen gesetzlich angeordneter und freiwilliger Schiedsgerichtsbarkeit betont ${ }^{57}$ - zum Ausdruck, dass sich schiedsgerichtliche Verfahren nicht unmittelbar an den Garantien des

51 EKMR, Nr. 1197/61, X/RFDA.

52 Zur grundsätzlichen Zulässigkeit, aber auch zu Grenzen des Verzichts vgl. unter D.

53 EKMR, Nrn. 8588/79 und 8589/79, Bramelid and Malmström/Sweden.

54 Ibid., Rdnr. 30.

55 EKMR, Nr. 10881/84, R/Switzerland, D.R. 51, S. 93 ff.

56 Ibid., S. $100 \mathrm{f}$.

57 Ibid., S. 100 mit Hinweis auf EKMR, Nrn. 8588/79 und 8589/79, Bramelid and Malmström/ Sweden. 
Art. 6 Abs. 1 EMRK messen lassen müssen; eine direkte Bindung an Art. 6 Abs. 1 EMRK bestehe daher aus ihrer Sicht nicht.

Der EGMR war sodann in der Rechtssache Suovaniemi/Finland erstmalig mit der Einordnung der privaten Schiedsgerichtsbarkeit in den Anwendungsbereich des Art. 6 Abs. 1 EMRK konfrontiert. Aufgrund von Unstimmigkeiten im Rahmen von Verhandlungen über die Auflösung eines Gesellschaftsvertrages wurde im konkreten Fall zur Klärung offener Rechtsfragen ein Schiedsgericht eingesetzt. Nach dem Schiedsspruch ersuchte die unterlegene Partei zunächst erfolglos Rechtsschutz vor nationalen Gerichten; schließlich wandte sie sich an den EGMR. Dort rügte sie die Verletzung des Art. 6 Abs. 1 EMRK, da die staatlichen Gerichte Schiedssprüche aufrechterhalten haben, obgleich nach Ansicht der Beschwerdeführer zwei Schiedsrichter nicht das nötige Maß an Unabhängigkeit und Unparteilichkeit aufwiesen. In seiner Entscheidung griff der EGMR die wesentlichen Grundsätze der Kommission auf, indem er feststellt:

„In deciding [...] the Court limits itself to the particular circumstances of the present case, which concerned arbitral proceedings. In doing this it takes account also of the applicable legislative framework for arbitration proceedings and the control exercised by the domestic courts within that framework." 58

Damit macht der EGMR deutlich, dass auch er eine unmittelbare Bindung der Schiedsgerichte an Art. 6 Abs. 1 EMRK ablehnt. Zugleich scheint es, dass der EGMR aber die Kompetenz beansprucht, die gesetzliche Ausgestaltung und gerichtliche Kontrolldichte schiedsgerichtlicher Verfahren in gewissem Umfang zu überprüfen. Diese mittelbare Überprüfung von Schiedssprüchen hat auch schon die Kommission angedeutet. In Jakob Boss Söhne $K G /$ Germany stellte die Kommission mit Blick auf die staatliche Verantwortlichkeit für Schiedssprüche im Lichte des Art. 6 Abs. 1 EMRK fest:

"[The waiver] does not mean, that the respondent State's responsibility is completely excluded as the arbitration award had to be recognized by the German courts and be given. The courts thereby exercised a certain control and guarantee as to the fairness and correctness of the arbitration proceedings which they considered to have been carried out in conformity with fundamental rights and in particular with the right of the applicant company to be heard."59

Zur Frage der staatlichen Verantwortlichkeit äußerte sich die Kommission auch in der Rechtssache Nordström/Netherlands. Der Beschwerdeführer rügte dort die Besetzung eines Schiedsgerichts, das aufgrund einer Schiedsklausel in einem ,joint-venture-agreement $t^{\text {“ }}$ zur Entscheidung im Streitfall ermächtigt worden war, da es einem Schiedsrichter an persönlicher Unabhängigkeit mangeln sollte. In ihrer Entscheidung wies die Kommission ebenfalls auf ihre zumindest mittelbare Überprüfungskompetenz schiedsgerichtlicher Verfahren hin, indem sie festhält:

„However, the Commission considers that account must be taken not only of the arbitration agreement between the parties and the nature of the private arbitration proceedings, but also of the legislative framework providing for such proceedings in order to determine whether the

58 EGMR, Nr. 31737/96, Suovaniemi and others/Finland.

59 EKMR, Nr. 18479/91, Jakob Boss Söhne KG/Germany. 
domestic courts retained some measure of control of the arbitration proceedings and whether this control has been properly exercised in the concrete case." ${ }^{\text {"60 }}$

Nach alledem lässt sich aus der Rechtsprechung der Konventionsorgane schließen, dass diese die private, echte Schiedsgerichtsbarkeit nicht als Adressat des Art. 6 Abs. 1 EMRK betrachten. ${ }^{61}$ Jedoch beanspruchen sowohl die EKMR als auch der EGMR eine gewisse Kontrolle der gesetzlichen Ausgestaltung des nationalen Schiedsrechts sowie des staatlichen Gerichtsverfahrens mit Blick auf die schiedsgerichtliche Entscheidung.

\section{Meinungsstand in der Literatur}

Die überwiegende Mehrheit der Autoren teilt die Ansicht der EKMR, des EGMR und der nationalen Gerichte und sieht die Schiedsgerichte nicht als unmittelbare Adressaten des Art. 6 Abs. 1 EMRK. ${ }^{62}$ Im Gegensatz zur zumeist apodiktischen Begründungsweise der Konventionsorgane wird dabei auf den Geltungsbereich völkerrechtlicher Verträge verwiesen. Denn diese binden nur die Staatsgewalt, nicht aber verpflichten völkerrechtliche Abkommen Private zur Einhaltung der in den Verträgen niedergelegten Rechte. Für die EMRK ergebe sich dies aus Art. 1 EMRK, wonach eben (nur) die Vertragsparteien allen ihrer Hoheitsgewalt unterstehenden Personen die in der Konvention bestimmten Rechte und Freiheiten zusichern.

Für die meisten Vertreter dieser fehlenden unmittelbaren Bindung an Art. 6 Abs. 1 EMRK hat dies indes nicht zugleich die völlige Bedeutungslosigkeit des Art. 6 Abs. 1 der Konvention zur Folge. Vielmehr befürworten diese eine mittelbare Bindung der Schiedsgerichte an Art. 6 Abs. 1 EMRK. ${ }^{63}$ Denn die Schnittstellen zwischen privater und staatlicher Justiz sollen gleichermaßen die Einbruchstellen für die Wertungen der EMRK darstellen. ${ }^{64}$ Anknüpfungspunkt einer ,mittelbaren oder indirekten Drittwirkung“ ist die Tatsache, dass der Schiedsspruch nicht in einen „rechtslosen Raum“ ergeht, sondern staatlichen

60 EKMR, Nr. 28101/95, Nordström/Netherlands.

61 In dem Verfahren vor der EKMR, Nr. 11960/86, Axelsson and others/Sweden, war ebenfalls ein privater Schiedsspruch Gegenstand des Verfahrens. Die EKMR überprüfte indes nur das den staatlichen Instanzenzug durchlaufende Verfahren nach der Entscheidung des Schiedsgerichts, nicht aber das Schiedsverfahren selbst.

62 Jarrosson, (Fn. 8), S. 576 ff.; Knoepfler, (Fn. 8), S. 469 f.; Briner/Schlabrendorff, (Fn. 45), S. 93; Haas, (Fn. 8), S. 76 f.; Geimer, in: Schlosser (Hrsg.), Integritätsprobleme im Umfeld der Justiz, 1994, S. 159 f.; Banifatemi, in: Amselek (Hrsg.), Mélanges Cohen-Jonathan, 2004, S. 184; Reiner, (Fn. 14), S. 60; Hess, in: Gottwald (Hrsg.), Effektivität des Rechtsschutzes vor staatlichen und privaten Gerichten, 2006, S. 164 f.; Lew/Mistelis/Kröll, Comparative international commercial arbitration, 2003, Kap. 5, Rdnr. 5-74; Samuel, (Fn. 19), S. 424; Schultz, in: Besson/Hottelier/ Werro, (Fn. 8), S. 51 f.; Rigozzi, (Fn. 21), S. 472 f.; Landrove, (Fn. 8), S. 91; Beys, in: YessiouFaltsi et al. (Hrsg.), Festschrift Fenge, 1996, S. 10. A.A. indes Bangert, in: Breitenmoser et al. (Hrsg.), Liber Amicorum für Wildhaber, 2007, S. 58; Wedam-Lukic, (Fn. 8), S. 16 ff.; wohl auch Moitry, Right to a Fair Trial and the European Convention on Human Rights - Some Remarks on the République de Guinée Case, Journal of International Arbitration 1989, S. 121.

63 Vgl. etwa Matscher, (Fn. 50), S. 241 f.; Samuel, (Fn. 19), S. 424; wohl auch Schiavetta, Does the Internet Occasion New Directions in Consumer Arbitration in the EU?, Journal of Information, Law and Technology 2004, Pkt. 5; Haas, (Fn. 8), S. 77; Schultz, (Fn. 62), S. 51 f.; Lambert, in: Mélanges Velu, 1992, S. 1289 f.

64 Haas, (Fn. 8), S. 77. 
Organen eine - wenngleich eingeschränkte - Überprüfungskompetenz verbleibt. § 1059 Abs. 2 Nr. 2b) ZPO etwa benennt als Grund für die Versagung der Wirksamkeit eines Schiedsspruchs im Rahmen einer Aufhebungsklage die Verletzung des ordre public. Zu den Bestandteilen des ordre public zählt unbestritten auch der sogenannte ordre public procédural (verfahrensrechtlicher ordre public). ${ }^{65}$ Die Anforderungen, die Art. 6 Abs. 1 EMRK an Gerichtsverfahren stellt, sind dann im Rahmen der Überprüfung dieses ordre public procédural zu berücksichtigen, womit das nationale Gericht etwaige Schiedssprüche, deren Anerkennung im Gegensatz zu Art. 6 Abs. 1 EMRK stehen würde, als nichtig zu befinden hat. ${ }^{66}$ In diesem Falle sind die Schiedsgerichte zumindest in indirekter Weise an die EMRK gebunden, da es ihre grundlegendste Aufgabe ist, Schiedssprüche zu erlassen, die im Einklang mit der Rechtsordnung des Sitzstaates stehen. ${ }^{67}$ Folglich müssen sich auch die Schiedsgerichte - sozusagen über den Umweg des ordre public - an den Anforderungen des Art. 6 Abs. 1 EMRK orientieren.

Für die Sicherstellung der Verfahrensgarantien des Art. 6 Abs. 1 EMRK ist nach dieser Ansicht dann aber entscheidend, in welchem Umfang der EGMR staatliche Entscheidungen, deren Gegenstand die Wirksamkeit eines Schiedsspruchs bzw. eines Schiedsverfahrens darstellt, tatsächlich kontrolliert. Bei der Ausgestaltung dieser Überprüfung macht die EMRK zunächst keine konkreten Vorgaben; die EKMR und der EGMR hingegen haben mehrfach betont, dass den Vertragsstaaten im Hinblick auf die Überwachungsfunktion ein weiter Ermessensspielraum zusteht. ${ }^{68}$ Dennoch geben die Konventionsorgane gewisse, allerdings sehr weitgefasste Rahmenbedingungen für die staatliche Kontrolle schiedsgerichtlicher Verfahren vor.

In Nordström/Netherlands stellte die Kommission zur Frage der Einwirkung des Staates zur Sicherung der Rechte in Art. 6 EMRK bei schiedsgerichtlicher Entscheidungsfindung fest:

„However, the Commission considers that account must be taken not only of the arbitration agreement between the parties and the nature of the private arbitration proceedings, but also of the legislative framework providing for such proceedings in order to determine whether the domestic courts retained some measure of control of the arbitration proceedings and whether this control has been properly exercised in the concrete case."69

Die Kommission deutet dabei zwei Problemkreise an. Zum einen die Anforderungen an das „wie“ der Kontrolle, d.h. die Ausgestaltung der gesetzlichen Rahmenbedingungen für die staatliche, gerichtliche Überwachung schiedsgerichtlicher Verfahren. Zum anderen die

65 Münch, in: MüKo-ZPO, 4. Aufl. 2013, § 1059, Rdnr. 42 ff.; Raeschke-Kessler, in: Prütting/Gehrlein (Hrsg.), ZPO, 5. Aufl. 2013, § 1059, Rdnr. 46.

66 Für die Schweiz vgl. ausdrücklich das BG v. 21.2.2008, 4A_370/2007 (unveröffentlicht).

67 In diesem Sinne Matscher, (Fn. 50), S. 237; Briner/Schlabrendorff, (Fn. 45), S. 93; Landrove, (Fn. 8), S. 92; Haas, (Fn. 8), S. 77; Robinson/Kasolowsky, (Fn. 14), S. 462.

68 Vgl. nur EGMR, Nr. 31737/96, Suovaniemi and others/Finland: „The Court considers that the Contracting States enjoy considerable discretion in regulating the question on which grounds an arbitral award should be quashed, since the quashing of an already rendered award will often mean that a long and costly arbitral procedure will become useless and that considerable work and expense must be invested in new proceedings. "Siehe auch EKMR, Nr. 28101/95, Nordström/ Netherlands.

69 Ibid.; ähnlich auch EGMR, Nr. 31737/96, Suovaniemi and others/Finland. 
Frage hinsichtlich des „ob“ der Kontrolle, d.h. der Umfang der richterlichen Kontrollkompetenz hinsichtlich der konkreten Überprüfung des Schiedsspruchs.

Entscheidend für die Konventionsorgane zur Frage des ,wie“ der Kontrolle ist eine Gesamtschau der rechtlichen Rahmenbedingungen (,legal framework"), in die das Schiedsverfahren eingebettet ist. ${ }^{70}$ Hierin zeigt sich der den Mitgliedstaaten zugestandene weite Ermessensspielraum bei der Frage des ,wie“ der Kontrolle. Denn aus der „Gesamtschautheorie“ ergibt sich auch, dass nicht jeder Verstoß gegen die Verfahrensgarantien des Art. 6 EMRK durch Schiedsgerichte die Vertragsstaaten aus der Sicht der EMRK verpflichtet, dem Schiedsspruch die Wirksamkeit zu versagen. ${ }^{71}$ So gibt die EMRK etwa nicht vor, ob die Ausübung staatlicher Kontrolle schon während des laufenden schiedsgerichtlichen Verfahrens oder aber erst nach dem Schiedsspruch erfolgen muss. ${ }^{72}$ Der EGMR hat auch entschieden, dass ein Verstoß gegen Art. 6 Abs. 1 EMRK ausscheidet, sofern der Beschwerdeführer die Möglichkeit der Befangenheitsrüge während des laufenden Prozesses verpasst hat und diese nun nachträglich erheben will. ${ }^{73}$ Ebenso wenig einen Verstoß gegen Art. 6 Abs. 1 EMRK stellt nach Ansicht der Kommission die Weigerung des Gerichts dar, einen Schiedsspruch wegen fehlender freiwilliger Verzichtserklärung aufzuheben, sofern das nationale Recht Möglichkeiten bereithält, im Vorfeld des Schiedsverfahrens die Freiwilligkeit der Schiedsabrede überprüfen zu lassen. ${ }^{74}$

Einen eingeschränkteren Ermessensspielraum gestehen die Vertreter einer mittelbaren Drittwirkung dem Konventionsstaat bei dem „ob“ der Kontrolle zu. Dort sind die Staaten verpflichtet, die Gewährleistung unverzichtbarer Verfahrensgarantien in Schiedsverfahren sicherzustellen. Dogmatischer Anknüpfungspunkt dafür ist die Tatsache, dass einzelne Rechte der EMRK nicht als bloße negative Abwehrrechte gegen den Staat zu verstehen sind, sondern dass diesen Rechten unter bestimmten Voraussetzungen darüber hinaus auch eine positive Funktion zukommen kann. ${ }^{75}$ Ein Anwendungsfall dieser Schutzpflichtdogmatik sei demnach die Verpflichtung des Staates, dafür Sorge zu tragen, dass in schiedsgerichtlichen Verfahren nicht gegen die in Art. 6 Abs. 1 EMRK niedergelegten, unverzichtbaren Verfahrensgarantien verstoßen werde. ${ }^{76}$ Gleichwohl hat weder die Kommission noch der EGMR jemals ausdrücklich eine aus Art. 6 Abs. 1 EMRK resultierende Schutzpflicht der Staaten für private schiedsgerichtliche Verfahren festgestellt. Ein Blick auf die jeweiligen Entscheidungen belegt vielmehr, dass die Konventionsorgane zwar eine Überprüfung der Verantwortlichkeit des Staates mit Blick auf nationale Gerichtsverfahren vornehmen. Zugleich sind aber die Staaten nicht dazu verpflichtet, unter Missachtung von Art. 6 Abs. 1 EMRK ergangene Schiedssprüche aufzuheben und damit die Garantien des Art. 6 Abs. 1 EMRK zwingend sicherzustellen. In $R /$ Switzerland etwa hielt die Kommission fest:

70 Siehe auch Haas, (Fn. 8), S. 81; Briner/Schlabrendorff, (Fn. 45), S. 99.

71 So ausdrücklich die EKMR, Nr. 28101/95, Nordström/Netherlands: „The Commission therefore considers that an arbitral award does not necessarily have to be quashed because the parties have not enjoyed all the guarantees of Article 6."

72 Im Einzelnen hierzu Briner/Schlabrendorff, (Fn. 45), S. 99 f.; Haas, (Fn. 8), S. 81 f.

73 Vgl. EGMR, Nr. 31737/96, Suovaniemi and others/Finland.

74 Vgl. EKMR, Nr. 24118/94, Hedland/Sweden.

75 Hierzu Krieger, in: Grote/Marauhn (Hrsg.), EMRK/GG, 2006, Kap. 6, Rdnrn. 21 ff., 103 ff.

76 Vgl. Matscher, (Fn. 50), S. 237; Haas, (Fn. 8), S. 77. 
„The Commission considers that the State cannot be held responsible for the arbitrators' action unless, and only insofar as, the national courts were required to intervene. "77

Noch deutlicher formulierte dies die Kommission in Nordström/Netherlands, indem sie judiziert, dass es aus Sicht der Konvention nicht erforderlich sei, dass schiedsgerichtliche Verfahren im Einklang mit Art. 6 Abs. 1 EMRK stehen. ${ }^{78}$ Konsequenterweise sah die Kommission die Niederlande auch nicht in der Pflicht, die Unparteilichkeit des Schiedsgerichts durchzusetzen. Auch in den Verfahren Molin/Turquie ${ }^{79}$ und Axelsson and others/ Sweden $^{80}$ überprüfte die EKMR nicht, ob die Dauer des schiedsgerichtlichen Verfahrens mit Art. 6 Abs. 1 EMRK vereinbar war. Wiederum stellte die Kommission fest, dass die Konventionsstaaten nur dann verantwortlich sind, wenn die staatlichen Gerichte verpflichtet waren, den Schiedsspruch nach nationalem Recht aufzuheben.

Dieser Linie hat sich der EGMR in der Rechtssache Suovaniemi/Finland im Wesentlichen angeschlossen, indem er anführt:

"The Court considers that the Contracting States enjoy considerable discretion in regulating the question on which grounds an arbitral award should be quashed."81

Folglich haben bislang weder die EKMR noch der EGMR eine positive Verpflichtung der Staaten zur Aufhebung von Schiedssprüchen, die nicht im Einklang mit Art. 6 Abs. 1 EMRK stehen, festgestellt. Dies unterstreicht auch der Vergleich mit der Rechtsprechung des EGMR zur verfahrensrechtlichen Schutzwirkung in klassischen staatlichen Gerichtsverfahren. Dort hat der EGMR mehrfach in ausdrücklicher Weise festgestellt, dass die Erfüllung einer Verpflichtung aus der Konvention ein positives Tun seitens des Staates erforderlich macht. ${ }^{82}$ Eine solche positive Handlungspflicht begründe eben auch Art. 6 Abs. 1 EMRK, etwa im Blick auf die Pflicht des Staates, seine Rechtspflege in der Weise auszugestalten, dass sie den Vorgaben des Rechts auf gerichtliches Gehör aus Art. 6

77 EKMR, Nr. 10881/84, R/Switzerland, D.R. 51, S. 102.

78 EKMR, Nr. 28101/95, Nordström/Netherlands: „It cannot be required under the Convention that national courts must ensure that arbitral proceedings have been in conformity with Article 6 of the Convention. The Commission therefore considers that an arbitral award does not necessarily have to be quashed because the parties have not enjoyed all the guarantees of Article 6, but each Contracting State may in principle decide itself on which grounds an arbitral award should be quashed."

79 EKMR, Nr. 23173/94, Molin/Turquie.

80 EKMR, Nr. 11960/86, Axelsson and others/Sweden.

81 EGMR, Nr. 31737/96, Suovaniemi and others/Finland.

82 Vgl. EGMR, Nr. 6289/73, Airey/Irland, Rdnr. 25 = EuGRZ 1979, 626 ff.: „Furthermore, fulfilment of a duty under the Convention on occasion necessitates some positive action on the part of the State; in such circumstances, the State cannot simply remain passive and there is [...] no room to distinguish between acts and omissions. The obligation to secure an effective right of access to the courts falls into this category of duty." Noch deutlicher EGMR, Nr.11371/85, Martins Moreira/Portugal, Rdnr. 60: „The Portuguese State is under a duty to provide them with appropriate means in relation to the objectives pursued so as to enable them to comply with the requirements of Article 6 para. 1.“ 
Abs. 1 EMRK genügt. ${ }^{83}$ Im Zusammenhang mit der Überprüfung schiedsgerichtlicher Entscheidungen haben die Konventionsorgane in keiner Weise von einer derartigen Pflicht $($, ,duty") der Mitgliedstaaten gesprochen, die sicherstellen soll, dass die Gerichte die Verfahrensgarantien des Art. 6 EMRK gewährleisten können.

Demnach lässt sich aus der Rechtsprechung der Konventionsorgane keine ausreichende Grundlage für die Annahme einer Schutzpflicht aus Art. 6 Abs. 1 EMRK für schiedsgerichtliche Verfahren entnehmen. Die Konventionsorgane untersuchen vielmehr, ob die nationalen Instanzen bei der Überprüfung des Schiedsspruchs die Garantien des Art. 6 Abs. 1 der Konvention berücksichtigt haben, nehmen diese Prüfung aber nur in dem Maße vor, als das nationale Recht eine derartige Prüfung überhaupt vorsieht. ${ }^{84}$ Damit ist das Vorliegen einer nationalen Regelung bezüglich der Überprüfung von Schiedsentscheidungen conditio sine qua non für die Überprüfungskompetenz des EGMR; ansonsten besteht kein Anknüpfungspunkt aus Sicht der Konvention, um die Einhaltung der Verfahrensrechte aus Art. 6 Abs. 1 EMRK zu überwachen. Selbst in den Fällen, die einen Anknüpfungspunkt für die Überprüfung durch den EGMR bieten, wird der Gerichtshof - in Anbetracht der bisherigen Rechtsprechung der Konventionsorgane, die sich im Wesentlichen durch äußerste Zurückhaltung gegenüber den Vertragsstaaten auszeichnet - wohl nur in krassen Ausnahmekonstellationen eine Verletzung von Art. 6 Abs. 1 EMRK überhaupt feststellen. ${ }^{85}$ Die Konventionsorgane stellen die Überprüfbarkeit des Art. 6 Abs. 1 EMRK damit faktisch in das Ermessen der Konventionsstaaten. Mit Blick auf die Bedeutung des Art. 6 Abs. 1 EMRK ist dies ein schwerlich akzeptables Ergebnis.

Insgesamt zeigt sich daher, dass die Theorie der mittelbaren Drittwirkung des Art. 6 Abs. 1 EMRK eine durchaus elegante Lösung im Spannungsfeld der Reichweite der Konventionsrechte und der Gewährleistung individueller Verfahrensrechte auch in der ,,privaten“ Gerichtsbarkeit darstellt. Zugleich aber mangelt es dieser Lösung an rechtspraktischer Substanz, denn sowohl die EKMR als auch der EGMR üben große Zurückhaltung im Rahmen der Überprüfung staatlicher Urteile über vorgelagerte schiedsgerichtliche Verfahren. Die „Theorie“ der mittelbaren Bindung ist folglich auch davon abhängig, in welchem Maße der EGMR seine Kontrollkompetenz bezüglich der Konventionsstaaten in schiedsgerichtlichen Verfahren wahrnimmt. Zieht sich der Gerichtshof aber weitgehend in seiner Funktion als überwachendes Organ zurück, bedingt dies zwangsläufig Lücken im Grundrechtsschutz. All dies führt zu einer Verkürzung existentieller Verfahrensrechte, die vor dem Hintergrund der grundlegenden Bedeutung von ,fair trials“ im internationalen Menschenrechtsschutz kaum hinnehmbar sind.

83 Vgl. EGMR, Nr. 6289/73, Airey/Irland, Rdnr. 25 = EuGRZ 1979, 626 ff.; EGMR, Nr. 14146/88, Muti/Italy, Rdnr. 15. Im Hinblick auf angemessene Verfahrensdauer siehe etwa EGMR, Nr. 8990/80, Guincho/Portugal, Rdnr. 38; EGMR, Nr. 10527/83, Milasi/Italy, Rdnr. 18. Eingehend zu verfahrens- und organisationsrechtlicher Schutzwirkung im Lichte der EMRK Krieger, (Fn. 75), Kap. 6, Rdnr. $103 \mathrm{ff}$.

84 Vgl. auch Bangert, (Fn. 62), S. 50.

85 Die Zurückhaltung der Rechtsprechungsorgane der EMRK betont auch Matscher, (Fn. 50), S. 243. 


\section{Stellungnahme - Zugleich ein Plädoyer für eine unmittelbare Bindung an Art. 6 Abs. 1 EMRK}

Diese lediglich indirekte Wirkung der EMRK in sportschiedsgerichtlichen Verfahren ist nicht nur wegen der geringen Kontrolldichte durch den EGMR im Hinblick auf die staatliche Überwachungsfunktion problematisch.

Nicht ganz geklärt ist auch der dogmatische Anknüpfungspunkt für eine mittelbare Drittwirkung des Art. 6 Abs. 1 EMRK. Angesichts der beschränkten Überprüfungskompetenz staatlicher Gerichte mit Blick auf schiedsgerichtliche Verfahren ist dies keineswegs unproblematisch. Denn Art. 6 Abs. 1 EMRK setzt voraus, dass das zu überprüfende Verfahren eine Streitigkeit in Bezug auf zivilrechtliche Ansprüche und Verpflichtungen zum Gegenstand hat. Das staatliche Gericht prüft den Schiedsentscheid aber gerade nicht mehr in inhaltlicher Hinsicht. ${ }^{86}$ Folglich ist - präzise betrachtet - schon der sachliche Anwendungsbereich des Art. 6 Abs. 1 EMRK nicht eröffnet, da das nationale Gericht, das zwecks Aufhebung des Schiedsspruchs angerufen wird, nicht über zivilrechtliche Ansprüche oder Verpflichtungen zu befinden hat; diese Aufgabe fällt vielmehr dem Sportschiedsgericht selbst zu. ${ }^{87}$

Darüber hinaus ist auch die Feststellung der Verantwortlichkeit des Staates nicht in jedem Fall unproblematisch, sofern die mittelbare Drittwirkung des Art. 6 Abs. 1 EMRK befürwortet wird. Der Anknüpfungspunkt für die Verantwortlichkeit des Vertragsstaates unter der Konvention ist unbestritten weniger territorialer Natur, sondern vielmehr mit der Ausübung von Hoheitsgewalt verbunden. ${ }^{88}$ Diese wird freilich primär auf eigenem Staatsgebiet ausgeübt, kann aber auch außerhalb des Territoriums zu Verletzungen einzelner Garantien der EMRK führen. Insgesamt kommt es aus Sicht der Konvention also darauf an, dass das potentielle Opfer durch die Ausübung von Hoheitsgewalt eines Konventionsstaates in einem in der EMRK garantierten Recht verletzt worden ist. Betrachtet man nun den Schiedsspruch als Akt ohne jegliche hoheitliche Wirkung, so wird nicht ganz deutlich, worin nun ein Anknüpfungspunkt für eine „mittelbare“ Prüfung zu sehen ist; allein der Umstand, dass das Schiedsgericht seinen Sitz in einem der Konvention angehörigen Staat hat, reicht meines Erachtens für eine Zurechnung an den Sitzstaat jedenfalls - in Anbetracht der oben dargestellten Anforderung der ,Jurisdiktionsgewalt“ - nicht aus. ${ }^{89}$

Zur Veranschaulichung soll folgender fiktiver sportrechtlicher Fall dienen. ${ }^{90}$ Angenommen, ein amerikanischer Sportverband sowie ein dem Verband angehöriger Sportler strei-

86 Vgl. Münch, (Fn. 65), § 1059, Rdnr. 7: „keine Inhaltskontrolle des Schiedsspruchs, nicht einmal in Gestalt einer Billigkeitsprüfung oder Willkürkontrolle." Das staatliche Gericht überprüft nur, ob die Anerkennung des Schiedsspruchs dem ordre public widerspricht. Für eine vergleichende Untersuchung mit Blick auf andere Konventionsstaaten vgl. Poudret/Besson, Comparative Law of International Arbitration, 2007, S. $801 \mathrm{ff}$.

87 Ebenso Bangert, (Fn. 62), S. 52; a.A. indes aufgrund einer aus Art. 6 Abs. 1 EMRK fließenden positiven Schutzpflicht Haas, (Fn. 8), S. 82.

88 Vgl. auch mit Hinweis auf EGMR-Rechtsprechung Röben, in: Grote/Marauhn, (Fn. 75), Kap. 5, Rdnr. $74 \mathrm{ff}$.

89 Vgl. auch Bangert, (Fn. 62), S. 53.

90 Ähnliche Konstellationen können freilich auch in Streitigkeiten ohne sportrechtlichen Bezug auftreten. Entscheidend ist letztlich die Tatsache, dass die Streitigkeit außerhalb des Anwendungsbereichs der Konvention angesiedelt ist. 
ten sich über eine nach amerikanischem Verbandsrecht erfolgte Maßnahme. Ist eine vereinsinterne Streitschlichtung nicht möglich, so wäre ein staatliches, amerikanisches Gericht zur Klärung offener Rechtsfragen berufen, womit freilich Art. 6 Abs. 1 EMRK keinerlei Bedeutung zukommt. Derogieren die Parteien jedoch die staatliche Justiz und bestimmen den CAS als Entscheidungsinstanz, so gerät das Verfahren nun in den Blickfang des Art. 6 Abs. 1 EMRK, da die Schweiz ein Konventionsstaat ist. Demnach ist allein der Umstand, dass das betreffende Schiedsgericht seinen Sitz in einem Mitgliedstaat der EMRK hat, für die Maßgeblichkeit des Art. 6 Abs. 1 EMRK verantwortlich, denn der Fall liegt offensichtlich außerhalb des Jurisdiktionsgebietes der EMRK. Sieht man nun das Schiedsgericht nicht unmittelbar an Art. 6 Abs. 1 EMRK gebunden, so ist kein Grund ersichtlich, warum nationale Gerichte oder der EGMR im Rahmen einer „mittelbaren“ Drittwirkung das Verfahren auf seine Vereinbarkeit mit Art. 6 Abs. 1 EMRK kontrollieren sollten. ${ }^{91}$

Probleme treten ferner auf, wenn nicht nur ein Konventionsstaat betroffen ist, sondern die Schiedsvereinbarung zwei oder noch mehr Vertragsstaaten beschäftigt hat. Erhebt etwa ein Sportler im Vorfeld einer CAS-Entscheidung Klage vor einem deutschen Gericht, so wird sich dieses - die Erhebung der Einrede vorausgesetzt - aufgrund $\S 1032$ Abs. 1 ZPO für unzuständig befinden und die Klage abweisen. Begehrt der Sportler nach dem CASUrteil die Aufhebung der Entscheidung vor dem Schweizer Bundesgericht und befindet dieses das Vorbringen für erfolglos, wird auch das Bundesgericht die Klage abweisen. Rügt der Beschwerdeführer etwa die fehlende Wirksamkeit der Schiedsabrede, so kann er nach der Theorie der mittelbaren Drittwirkung sowohl Deutschland - denn auch das deutsche Gericht musste über die Wirksamkeit der Schiedsabrede befinden - als auch die Schweiz vor dem EGMR verantwortlich machen. Angenommen, die Schiedsvereinbarung ist tatsächlich unwirksam, wäre nach der Theorie der mittelbaren Drittwirkung konsequenterweise auch Deutschland seiner Schutzpflicht aus Art. 6 Abs. 1 EMRK nicht nachgekommen, da legislative und judikative Schutzvorkehrungen nicht ausreichend waren, die Konventionsverletzung zu verhindern, obwohl die eigentliche Verletzungshandlung einem anderen Konventionsstaat zurechenbar ist. Sinnvoller wäre es vor diesem Hintergrund, durch die unmittelbare Bindung an Art. 6 Abs. 1 EMRK den Staat verantwortlich zu machen, dessen nationales Recht den Schiedsspruch erst ermöglicht hat.

\section{Der Schiedsrichter - ein funktionaler Hoheitsträger im Lichte der EMRK}

Die Ungereimtheiten dieser von der Rechtsprechung und der Literatur bevorzugten „,mittelbaren Bindung" der Schiedsgerichte an Art. 6 Abs. 1 EMRK rühren auch daher, dass der Grundsatz der (fehlenden) Verpflichtung Privater durch völkerrechtliche Verträge in diesem Zusammenhang nur bedingt betroffen ist. Unbestritten verpflichten völkerrechtliche Verträge grundsätzlich zwar nur die Staaten, nicht aber auch Private. ${ }^{92}$ Dies führt dazu,

91 Ähnlich auch Bangert, (Fn. 62), S. 53.

92 Vgl. hierzu nur Kälin/Künzli, Universeller Menschenrechtsschutz, 2009, S. 94 ff. Für die EMRK vgl. Röben, (Fn. 88), Kap. 5, Rdnr. 101; Frowein, in: ders./Peukert (Hrsg.), EMRK-Kommentar, 2009, Art. 1, Rdnr. 16. Eine partielle Ausnahme von diesem Grundsatz bilden einzelne Bestimmungen des Unionsrechts, dessen Sonderstellung in diesem Zusammenhang aber in der Supranationalität der Union gründet. 
dass sich Individuen untereinander nicht auf die Verletzung von Menschenrechten und damit auch auf eine etwaige Verletzung der EMRK berufen können.$^{93}$ Es darf nun aber nicht übersehen werden, dass die Schiedsgerichtsbarkeit eine Sonderform mit Blick auf diesen Grundsatz darstellt. Zwar lässt sich die schiedsrichterliche Zuständigkeitsbegründung für die Streitentscheidung zunächst noch unproblematisch auf die privatrechtliche Vereinbarung der Partien zurückführen. Dieses Verhalten der Partien wirft gewiss keinerlei (unmittelbare) Berührungspunkte mit der EMRK auf. Hiervon zu trennen ist allerdings die Entscheidung der Schiedsrichter selbst, die im Lichte der Konvention nicht ohne weiteres als Akt rein privatrechtlicher Natur bezeichnet werden kann. Gerade dies wird weithin von denjenigen Stimmen übersehen, die dem Schiedsspruch keine hoheitliche Wirkung zugestehen wollen. Denn diese untersuchen die Frage der hoheitlichen Tätigkeit häufig einzig unter dem Gesichtspunkt der Benennung der Schiedsrichter bzw. der Einsetzung des Schiedsgerichts, ohne die Wirkung der Entscheidung selbst zu diskutieren, die im Lichte internationaler Menschenrechtsverbürgungen durchaus einen Anknüpfungspunkt für die hoheitliche Natur von Schiedssprüchen bieten kann. ${ }^{94}$ Denn wenn die Schiedsrichter ihre Befugnis allein aus der Schiedsvereinbarung ableiten sollen, ${ }^{95}$ so kann das die Wirkung, die der Staat einem Schiedsentscheid zugesteht, nicht ausreichend begründen.

Der Schiedsrichter tritt, durch staatliche Vorschriften legitimiert, ${ }^{96}$ an die Stelle staatlicher Rechtsprechung, womit auch die Pflicht verbunden ist, eine mit der Rechtsordnung des Sitzstaates übereinstimmende Entscheidung einer Rechtsstreitigkeit zu fällen. Damit zeigt sich zum einen, dass das Schiedsgericht nicht etwa in einem vom staatlichen Recht losgelösten Rechtsraum agiert, sondern seine Möglichkeit zur Konstituierung in zumindest mittelbarer Weise auf gesetzliche Vorschriften zurückzuführen ist. Zum anderen wird die Entscheidung über die von Art. 6 Abs. 1 EMRK erfassten zivilrechtlichen Ansprüche und Verpflichtungen von staatlicher Seite als rechtskräftig anerkannt und kann sogar - in materiell-rechtlicher Hinsicht kaum geprüft - als Grundlage von Vollstreckungsmaßnahmen dienen. Damit übernimmt der Schiedsrichter eine Funktion, die grundsätzlich dem Staate eigen und vorbehalten ist; der Schiedsspruch ist daher ein unter bestimmten Voraussetzungen vorgesehener Akt erkennender Rechtsprechung. ${ }^{97}$ Dieser Bereich ist nun eine staatliche Aufgabe, womit sich veranschaulichen lässt, dass der Schiedsspruch im Lichte der EMRK nicht bar jeglichen hoheitlichen Charakters ist.

93 Für die EMRK ausdrücklich in EGMR, Nr. 24699/94, VGT Verein gegen Tierfabriken/Switzerland, Rdnr. 46: „The Court does not consider it desirable, let alone necessary, to elaborate a general theory concerning the extent to which the Convention guarantees should be extended to relations between private individuals inter se."

94 Jüngst etwa Spohnheimer, Gestaltungsfreiheit bei antezipiertem Legalanerkenntnis des Schiedsspruchs, 2010, S. 7 f.; Courvoisier, Anwendbares Recht vor internationalen Schiedsgerichten, 2005, S. 19. Vgl. ferner Schlosser, Das Recht der internationalen privaten Schiedsgerichtsbarkeit, 2. Aufl. 1989, Rdnr. 45 ff.; ders., in: Stein/Jonas (Hrsg.), ZPO, Bd. 9, 2002, vor § 1025, Rdnr. 2; eingehend auch Solomon, Verbindlichkeit von Schiedssprüchen in der internationalen privaten Schiedsgerichtsbarkeit, 2007, S. 326 ff. mit umfangreichen Nachweisen auf S. 322.

95 Vgl. nur Schlosser, in: Stein/Jonas, (Fn. 94), vor § 1025, Rdnr. 2.

$96 \S \S 1025 \mathrm{ff}$. ZPO.

97 Im Ergebnis auch Bangert, (Fn. 62), S. 56 f.; ähnlich Besson, (Fn. 17), S. 402; Ambrose, (Fn. 23), S. 485; Wedam-Lukic, (Fn. 8), S. 19. 
Nun mag eingewendet werden, dass der Schiedsrichter sich im Grunde nicht als Träger hoheitlicher Gewalt sieht. ${ }^{98}$ Dies ist zumindest insoweit zutreffend, als die Schiedsgerichte tatsächlich keinerlei staatliche Zwangsgewalt ausüben oder anordnen können; die zwangsweise Durchsetzung eines Schiedsspruchs ist dem Staat vorbehalten und nicht Aufgabe der Schiedsgerichte. Damit ist indes noch nicht zwangsläufig verbunden, dass dem Schiedsspruch im Anwendungsbereich der Konvention kein hoheitlicher Charakter inhärent wäre. Die Rechtsprechungsfunktion eines Staates beschränkt sich nicht allein auf die Ausübung staatlicher Zwangsgewalt zur Durchsetzung der Entscheidungen. Vorgelagert, und ebenfalls von der Rechtsprechungsfunktion erfasst, ist die Rechtsanerkennung, die erst die etwaige anschließende Rechtsdurchsetzung ermöglicht. Gerade dieser Teilbereich der Rechtsprechungsfunktion fällt nun aber in den Aufgabenbereich des Schiedsgerichts; es entscheidet über die materielle Rechtslage an Stelle eines üblicherweise dazu berufenen staatlichen Gerichts. Damit partizipieren private Schiedsgerichte sehr wohl an der Ausübung staatlicher Hoheitsgewalt; ihre Teilhabe ist aber eben nur rein judikativer Natur. ${ }^{99}$

Im Rahmen funktionaler Hoheitsgewalt, also der Erfüllung staatlicher Aufgaben durch Private, wird nun aber im Lichte der EMRK eine staatliche Delegation oder Verleihung hoheitlicher Befugnisse an Private verlangt, um die Verantwortlichkeit der Mitgliedstaaten zu begründen. ${ }^{100}$ Sieht man die Rechtsprechungsfunktion als Anknüpfungspunkt für eine hoheitliche Tätigkeit, findet eine solche unmittelbare Übertragung von Hoheitsgewalt - wie beispielsweise bei der staatlich angeordneten Schiedsgerichtsbarkeit - zumindest auf direktem Wege tatsächlich nicht statt. Gesteht das nationale Recht dem Schiedsrichter das Recht zu, eine Entscheidung in der Sache zu treffen und diese Entscheidung von staatlicher Seite als endgültig und vollstreckbar anzuerkennen, ist darin vielleicht nicht unbedingt die klassische Form der „Übertragung von Hoheitsgewalt“" zu sehen. Ob es darauf aber letztlich ankommen kann, mag bezweifelt werden, denn zumindest in funktioneller Hinsicht kommt der Entscheidung der privaten Schiedsgerichte eine ähnliche Bedeutung zu wie der eines staatlich angeordneten Schiedsgerichts. Diese Tatsache macht die Besonderheit eines privaten Schiedsspruchs aus. Die Schiedsentscheidung begründet sich aus der im nationalen Recht niedergelegten Möglichkeit der privaten Schiedsschlichtung und ist zugleich das Ergebnis der staatlichen Rechtsordnung, nach deren Maßgabe das Schiedsgericht erst verfahren kann. Eben diese Stellung im nationalen, innerstaatlichen Rechtsgefüge des Sitzstaates ermöglicht es, den Schiedsspruch im Lichte der EMRK als Hoheitsakt zu qualifi-

98 So auch für das nationale Recht der BGH, vgl. BGHZ 51, 255 (258); 54, 392 (395); 65, 59 (61): „Ein Schiedsgericht übt zwar keine öffentliche Gewalt aus, ist aber gleichwohl materiell Rechtsprechung." Ebenso wird in der deutschen Literatur dem Schiedsspruch mehrheitlich der hoheitliche Charakter versagt, vgl. nur Schlosser, in: Stein/Jonas, (Fn. 94), vor § 1025, Rdnr. 2; von Hoffmann, Internationale Handelsschiedsgerichtsbarkeit, 1970, S. 37; jüngst Steiner, Das Verhältnis von Schiedsgerichtsbarkeit und staatlicher Gerichtsbarkeit, SchiedsVZ 2013, S. 19. Siehe auch mit umfassenden weiteren Nachweisen Solomon, (Fn. 94), S. 322 f. Für die Schweiz vgl. nur Rüede/Hadenfeldt, Schweizerisches Schiedsgerichtsrecht nach Konkordat und IPRG, 2. Aufl. 1993, S. 3.

99 Ebenso Bangert, (Fn. 62), S. 56; siehe ferner Nobel, in: Forstmoser/Schluep (Hrsg.), Festschrift Meier-Hayoz, 1982, S. 248. Ähnlich auch Habscheid, Das neue Recht der Schiedsgerichtsbarkeit, JZ 1998, S. 446.

100 EGMR, Nr. 13134/87, Castello-Roberts, Rdnr. 27. Hierzu auch Röben, (Fn. 88), Kap. 5, Rdnr. 73; Frowein, (Fn. 92), Art. 1, Rdnr. 14; Grabenwarter/Pabel, (Fn. 11), § 17, Rdnr. 6. 
zieren; ob dieser Umstand nun als Delegation bezeichnet werden kann oder nicht, ist letztlich aus Sicht der EMRK meines Erachtens nicht entscheidend. ${ }^{101}$

\section{Sportspezifische Argumente}

Insbesondere im internationalen sportrechtlichen Kontext lässt sich die Qualität eines Schiedsspruches als faktischen „Hoheitsakt“ trefflich veranschaulichen. Hat der CAS etwa ein für einen deutschen Sportler nachteiliges Urteil gefällt, so kann dieser nur in der Schweiz, nicht aber auch in Deutschland die Aufhebung des Schiedsspruchs beantragen. Das schweizerische Recht sieht aber zusätzlich noch die Möglichkeit vor, durch eine ausdrückliche Erklärung in der Schiedsvereinbarung die Anfechtung ausländischer Schiedsentscheide vollständig auszuschließen, vgl. Art. 192 Abs. 1 IPRG. ${ }^{102}$ Die letzte Möglichkeit für den deutschen Sportler, die Wirksamkeit des Schiedsspruchs in irgendeiner Weise anzugreifen, verbleibt sodann in der Versagung der Anerkennung und Vollstreckung der Schiedsentscheidung, vgl. $\S 1061$ Abs. 1 ZPO in Verbindung mit Art. V UNÜ. Aber auch die wird dem Sportler verwehrt bleiben, wenn der CAS über die Rechtmäßigkeit von Dopingsperren zu befinden hat, denn in diesen Fällen wird ein dem Schiedsspruch nachfolgender Antrag auf Anerkennung oder Vollstreckung niemals gestellt werden. Der Schiedsspruch des CAS ist auf diese Weise der Kontrolle von staatlicher Seite faktisch entzogen; der Unterschied zu sonstigem privaten Verhalten tritt hierbei evident zu Tage.

Auch die Tatsache, dass inzwischen in vielen internationalen Sportverbandssatzungen in zunehmendem Maße Schiedsklauseln ,,angeordnet“" werden, zeigt, dass sich zumindest die internationale Sportschiedsgerichtsbarkeit in funktioneller Hinsicht der staatlich angeordneten Schiedsgerichtsbarkeit annähert. Denn letztlich macht es aus Sicht des Sportlers keinen Unterschied, ob er infolge staatlich oktroyiertem Schiedszwang - der die unmittelbare Bindung an Art. 6 Abs. 1 EMRK auslöst - oder aufgrund wirtschaftlicher Abhängigkeit von privater Verbandsseite gezwungen wird, auf sein verfassungs- und völkerrechtlich garantiertes Recht auf Zugang zu einem staatlichen Gericht zu verzichten. Nicht nur dieses Beispiel zeigt, dass sich das Verhältnis Verband und Sportler allmählich dem Verhältnis Staat und Bürger angleicht.

Das System der Schiedsgerichtsbarkeit beruht unter anderen aber auch auf dem Prinzip der Waffengleichheit. Gerade das Subordinationsverhältnis im internationalen Sport höhlt dieses Prinzip weitestgehend aus. Schon vor diesem Hintergrund zeigt sich, dass internationale Sportschiedsgerichte Fälle entscheiden zu haben, die sich nicht mit globalen Wirt-

101 Siehe auch Bangert, (Fn. 62), S. 56; a.A. aber etwa Münzberg, Schranken der Parteivereinbarung in der privaten internationalen Schiedsgerichtsbarkeit, 1970, S. 24.

102 Eine vergleichbare Bestimmung enthält auch das schwedische Zivilprozessrecht, vgl. § 51 des Swedish Arbitration Act (SFS 1999:116) oder der belgische Code Judiciaire, vgl. Art. 1718. Zu weiteren Beispielen aus dem europäischen Rechtsraum siehe Briner/Schlabrendorff, (Fn. 45), S. 100. Das BG hat aber entschieden, dass dieser im Voraus erklärte Verzicht im Lichte der Besonderheiten der Sportschiedsgerichtsbarkeit nicht möglich ist, vgl. BG v. 23.3.2007 = SchiedsVZ 2007, 330 ff. Dennoch zeigt diese Konstellation die grundsätzliche Auswirkung schiedsrichterlicher Entscheidungen; auch kann unter Umständen ein Gericht zu einem anderen Ergebnis kommen, insbesondere da diese Verzichtsmöglichkeit in einigen EMRK-Vertragsstaaten anerkannt ist. 
schaftsstreitigkeiten vor Schiedsgerichten vergleichen lassen, in welchen sich die Parteien in der Regel auf Augenhöhe begegnen. Vielmehr kennzeichnet internationale Sportschiedsverfahren die Tatsache, dass ohne das Subordinationsverhältnis sowie der Eigengesetzlichkeit und Internationalität des Sports die Auseinandersetzung niemals vor einem Schiedsgericht, sondern vor einem regulären staatlichen Gericht stattfinden würde. Die zunehmende Verlagerung sportrelevanter internationaler Konfliktlösung darf nun eben nicht dazu führen, dass die wesentlichen Garantien des Art. 6 Abs. 1 EMRK nicht mehr auf unmittelbarem Wege gewährleistet werden können, da staatliche Gerichte aufgrund internationaler Sportverbandssatzungen nicht mehr angerufen werden (können).

\section{Zwischenergebnis}

Angesichts der voranstehenden Erwägungen bietet es sich aus der Sicht der EMRK an, schiedsrichterliche Entscheidungen als ein dem Staat zurechenbaren Hoheitsakt zu qualifizieren, womit das schiedsrichterliche Verfahren unmittelbar an den Garantien des Art. 6 Abs. 1 EMRK gemessen werden kann. Soweit also nationale Rechtsordnungen Schiedsgerichten das Recht einräumen, staatliche Funktionen wahrzunehmen, weist die staatliche Zuständigkeitsordnung ihnen damit auch die Aufgabe zu, die grundsätzlich den Staat betreffenden Pflichten aus der Konvention zu genügen. ${ }^{103}$ Trägt folglich ein Sportschiedsgericht den Verfahrensgarantien des Art. 6 Abs. 1 EMRK nicht Rechnung, so stellt dies eine unmittelbare Verletzung der Konvention dar.

\section{Umfang der Bindung an Art. 6 Abs. 1 EMRK}

Sind die Schiedsgerichte unmittelbar an Art. 6 Abs. 1 EMRK gebunden, verbleibt die Frage, welche Garantien des Art. 6 Abs. 1 EMRK für schiedsgerichtliche Verfahren von Bedeutung sind, aber auch, ob und in welchem Umfang auf diese Garantien verzichtet werden kann.

\section{Verfahrensgarantien des Art. 6 Abs. 1 EMRK}

Artikel 6 EMRK bildet eine wesentliche Ausprägung des in der Konvention zum Ausdruck kommenden rechtsstaatlichen Prinzips. ${ }^{104}$ Die dort garantierten Rechte sollen sicherstellen, dass gerichtliche Verfahren im Geltungsbereich der Konvention rechtsstaatlichen (Mindest-)Anforderungen genügen. Vor diesem Hintergrund sind diese Garantien auch für schiedsgerichtliche Verfahren relevant. ${ }^{105}$ Dazu gehören zunächst das Recht auf Zugang zu einem Gericht sowie die Öffentlichkeit und Mündlichkeit des Verfahrens. Ein weiteres fundamentales Recht, das sowohl staatliche als auch schiedsgerichtliche Verfahren gewährleisten müssen, ist die Unabhängigkeit und Unparteilichkeit der zur Entscheidung be-

103 Ebenso Bangert, (Fn. 62), S. 56 f.

104 Grabenwarter/Pabel, (Fn. 11), §24, Rdnr. 2.

105 Im Einzelnen Matscher, (Fn. 50), S. 236 f.; Briner/Schlabrendorff, (Fn. 45), S. 93 ff.; Reiner, (Fn. 14), S. 62. 
rufenen Personen. Damit korrespondiert die Garantie der richtigen Zusammensetzung der Schiedsrichter. Weiterhin von Bedeutung ist der Anspruch des Einzelnen auf ein faires Verfahren, insbesondere auf rechtliches Gehör. Letztlich kann sich auch vor einem Schiedsgericht das Verfahren über einen langen Zeitraum erstrecken, womit das Recht auf eine Entscheidung innerhalb einer angemessenen Frist betroffen sein kann. Eine Berufung auf die Unschuldsvermutung gemäß Art. 6 Abs. 2 EMRK mit Blick auf ein sportschiedsgerichtliches Verfahren kommt allerdings nicht in Betracht. Denn dieses Recht gilt, wie schon der Wortlaut zeigt, nur im Rahmen einer Strafanklage, ${ }^{106}$ nicht aber findet Art. 6 Abs. 2 EMRK auf ein schiedsgerichtliches Verfahren Anwendung. Deshalb kann auch das Vorbringen Pechsteins in dem noch anhängigen Verfahren gegen die Schweiz, dass das Verfahren vor dem CAS den Grundsatz der Unschuldsvermutung verletze, ${ }^{107}$ nicht erfolgreich sein.

\section{Verzicht auf die Verfahrensgrundrechte des Art. 6 Abs. 1 EMRK}

Sind diese Garantien grundsätzlich ebenfalls im Rahmen schiedsgerichtlicher Verfahren von Bedeutung, müssen dennoch die Besonderheiten der Sportschiedsgerichtsbarkeit angemessen berücksichtigt werden. Denn in einigen Fällen ist dem Schiedsverfahren der gleichzeitige Verzicht auf einzelne Rechte der EMRK inhärent. So zielen gerade verschiedene Gründe für den Entschluss zur schiedsgerichtlichen Klärung auf den Verzicht von in Art. 6 Abs. 1 EMRK garantierten Verfahrensrechten ab. In diesem Zusammenhang hat der EGMR zu Recht festgestellt, dass die Verfahrensgarantien des Art. 6 Abs. 1 der Konvention keine absolute Geltung beanspruchen. ${ }^{108}$ Dies bedeutet allerdings nicht zugleich, dass auf diese Rechte nun auch verzichtet werden kann. Die Absolutheit einer Rechtsnorm ist ganz grundsätzlich von der Frage der Zulässigkeit eines Verzichts auf diese zu trennen. Entscheidend ist vielmehr, ob die Rechte aus Art. 6 EMRK disponibel sind, d.h. der Einzelne die Befugnis hat, auf die dort niedergelegten Rechte zu verzichten. Zu dieser Frage hat die Kommission Stellung bezogen und sich grundsätzlich für einen Verzicht einzelner aus Art. 6 EMRK resultierender Rechte ausgesprochen, indem sie festhält:

„Lastly, there is nothing in the Convention to prevent a person from renouncing the exercise of certain rights guaranteed under Article 6, paragraph I, in the case of a dispute involving civil rights and obligations, provided that the person's decision is taken freely and without coercion. " 109

106 Zum Anwendungsbereich siehe Meyer-Ladewig, Europäische Menschenrechtskonvention, 3. Aufl. 2011, Art. 6, Rdnr. $21 \mathrm{ff}$.

107 EGMR, Nr. 67474/10, Pechstein/Suisse.

108 EGMR, Nr. 9006/80, Lithgow and others/UK, Rdnr. 194a: „The right of access to the courts secured by Article 6 para. 1 is not absolute but may be subject to limitations."

109 EKMR, Nrn. 8588/79 und 8589/79, Bramelid and Malmström/Sweden; später auch EKMR, Nr. 10881/84, R/Switzerland; EKMR, Nr. 23173/94, Molin/Turquie; EKMR, Nr. 28101/95, Nordström/Netherlands. 
Auch der EGMR betont die grundsätzliche Zulässigkeit des Verzichts:

,$[A]$ waiver, which has undeniable advantages for the individual concerned as well as for the administration of justice, does not in principle offend against the Convention." ${ }^{110}$

Deshalb ist es grundsätzlich aus Sicht der EMRK auch nicht zu beanstanden, wenn die Parteien aufgrund einer wirksamen Schiedsvereinbarung auf einzelne in Art. 6 Abs. 1 EMRK niedergelegte Verfahrensgrundrechte verzichten. Aus der bisherigen Rechtsprechung der Konventionsorgane lassen sich folgende drei Voraussetzungen für den wirksamen Verzicht auf Verfahrensrechte des Art. 6 Abs. 1 EMRK erschließen. Der Verzicht muss zunächst auf freiwilliger Basis ${ }^{111}$ und in eindeutiger ${ }^{112}$ Weise erfolgen. Darüber hinaus dürfen dem Verzicht keine öffentlichen Interessen oder Rechte anderer entgegenstehen; ${ }^{113}$ denn der Einzelne darf nicht über Rechte disponieren, über die er nicht die ausschließliche Rechtsmacht innehat. ${ }^{114}$ Schließlich muss die Verzichtserklärung in Kenntnis der daraus resultierenden Umstände und Konsequenzen für die Parteien abgegeben worden sein:

,[the waiver] must be attended by minimum guarantees commensurate with the importance of the right waived. "115

Diese Voraussetzungen implizieren zugleich, dass die EMRK keinen „Totalverzicht“116 auf die Verfahrensrechte in schiedsgerichtlichen Verfahren zulassen will; beim Abschluss der Schiedsvereinbarung ist vielmehr nur ein ,„partieller Verzicht“ auf die Verfahrensgrundsätze des Art. 6 Abs. 1 EMRK zulässig. ${ }^{117}$ In diesem Sinne entschied auch der EGMR in Suovaniemi/Finland, indem er feststellt, dass

"there is no doubt that a voluntary waiver of court proceedings in favour of arbitration is in principle acceptable from the point of view of Article 6. Even so, such a waiver should not necessarily be considered to amount to a waiver of all the rights under Article 6."118

110 EGMR, Nr. 6903/75, Deweer/Belgium, Rdnr. 49; siehe ferner EGMR, Nr. 9006/80, Lithgow and others/UK, Rdnr. 194a; EGMR, Nr. 31737/96, Suovaniemi and others/Finland; zuletzt auch EGMR, Nr. 53146/99, Hurter/Suisse, Rdnr. 28.

111 Vgl. EKMR, Nr. 6903/75, Deweer/Belgium, Rdnr. 49.

112 „The waiver must be established in an unequivocal manner". Vgl. EKMR, Nr. 11960/86, Axelsson and others/Sweden; EGMR, Nr. 11662/85, Oberschlick/Austria, Rdnr. 51; EGMR, Nr. 10802/84, Pfeifer and Plankl/Austria, Rdnr. 37.

113, [A waiver] must not run counter to any important public interest ${ }^{“}$. Vgl. EMRK, Nr. 11960/86, Axelsson and others/Sweden; ebenso EGMR, Nr. 11855/85, Hakansson and Sturesson/Sweden, Rdnr. 66; zuletzt auch EGMR, Nr. 53146/99, Hurter/Suisse, Rdnr. 28.

114 So schon Jellinek, System der subjektiven öffentlichen Rechte, 2. Aufl. 1919, S. 340. Im Hinblick auf die EMRK vgl. ferner Grabenwarter/Pabel, (Fn. 11), § 18, Rdnr. 33.

115 Vgl. EGMR, Nr.31737/96, Suovaniemi and others/Finland; vgl. zur Begrifflichkeit auch Robinson/Kasolowsky, (Fn. 14), S. 464.

116 Matscher, (Fn. 50), S. 229. Vgl. auch EKMR, Nr. 11960/86, Axelsson and others/Sweden.

117 Ebenso Haas, (Fn. 8), S. 78; Besson, (Fn. 17), S. 400; Briner/Schlabrendorff, (Fn. 45), S. 91; Knoepfler, (Fn. 8), S. 470; Poncet/Cambi Favre-Bulle, (Fn. 23), S. 670. A.A. Monheim, Sportlerrechte und Sportgerichte im Lichte des Rechtsstaatsprinzips, 2006, S. 102, der den Verzicht grundsätzlich für nicht haltbar ansieht.

118 EGMR, Nr. 31737/96, Suovaniemi and others/Finland. 
Weiter stellt der EGMR fest, dass

„a waiver may be permissible with regard to certain rights but not with regards to certain others. A distinction may have to be made even between different rights guaranteed by Article 6." $" 119$

In der Folge führt der EGMR zwar aus, dass auf das Recht auf eine öffentliche Verhandlung in schiedsgerichtlichen Verfahren grundsätzlich verzichtet werden kann; im Übrigen lässt der EGMR aber im Argen, auf welche Rechte des Art. 6 Abs. 1 EMRK sich der Verzicht beziehen kann und welche Kriterien für die Unterscheidung verzichtbarer und unverzichtbarer Verfahrensgarantien maßgeblich sind.

Vor diesem Hintergrund stellt sich daher die Frage, auf welche in Art. 6 Abs. 1 EMRK niedergelegten Verfahrensrechte sich dieser ,,partielle Verzicht“ erstreckt. Unbestritten verzichten die Parteien durch die Schiedsabrede auf das in Art. 6 Abs. 1 EMRK statuierte Recht auf Zugang zu einem staatlichen Gericht. ${ }^{120}$ Ebenso wird den Parteien in der Regel daran gelegen sein, ihre Sache unter Ausschluss der Öffentlichkeit auf vertraulichem Wege zu verhandeln; dies gilt insbesondere im Hinblick auf Wirtschaftsstreitigkeiten globaler Konzerne, aber auch mit Blick auf sportschiedsgerichtliche Entscheidungen. Angesichts dieses Umstands wäre es grundsätzlich unsinnig, Schiedssprüche wegen des Verstoßes gegen den Grundsatz der Öffentlichkeit aus Sicht der EMRK zu beanstanden, zumal die mit diesem Grundsatz verbundenen Zwecke im Rahmen der Schiedsgerichtsbarkeit schon durch die Bestimmung der Schiedsrichter erfolgt. ${ }^{121}$ Denn im Gegensatz zu Verfahren vor staatlichen Gerichten, wo der Öffentlichkeitsgrundsatz das Vertrauen der Beteiligten in ein rechtsstaatliches Verfahren sicherstellt, ist dieses Vertrauen in die Entscheidungsfindung im Schiedsverfahren unmittelbar an die Auswahl der Schiedsrichter geknüpft. ${ }^{122}$ Auch der EGMR sieht im Übrigen nicht nur in der Entscheidung Suovaniemi/Finland, sondern auch unabhängig von schiedsgerichtlichen Zusammenhängen das Recht auf ein öffentliches Verfahren nicht als ein unverzichtbares Recht an. ${ }^{123}$ Gleichwohl mag es in besonders gelagerten Konstellationen, insbesondere im Rahmen von öffentlichkeitswirksamen Dopingfällen, im Einzelfall ein Bedürfnis für den betroffenen Sportler geben, ein öffentliches Verfahren zu verlangen. Dieses Anliegen ist unmittelbar mit dem Rehabilitationsinteresse des Sportlers verbunden, dass auch in der Öffentlichkeit eine unter Umständen rechtswidrige Dopingsperre als solche wahrgenommen wird. Damit wird sich in diesen Fällen der (vorherige) Abschluss einer Schiedsvereinbarung nicht immer auch auf den Verzicht auf den Öffentlichkeitsgrundsatz erstrecken. Deshalb darf das Sportschiedsgericht, um seinen

119 Ibid.

120 Fraglich ist aber häufig, ob sie dies freiwillig tun. Vgl. hierzu die Ausführungen unter D.III.

121 In diesem Sinne BG v. 10.2.2010, 4A_612/2009, Rdnr. 4.1. Siehe ferner Moitry, (Fn. 62), S. 121; Landrove, (Fn. 8), S. 85; Frumer, La renonciation aux droits et libertes: la Convention europeenne des droits de l'Homme a l'epreuve de la volonte individuelle, 2001, S. 118; Matscher, (Fn. 50), S. 237. Offen lassend Steiner, (Fn. 98), S. 19.

122 Haas, (Fn. 8), S. 78.

123 Vgl. etwa EGMR, Nr. 14518/89, Schuler-Zgraggen/CH=EuGRZ 1996, 604, Rdnr. 58. Zu weiteren zulässigen Beschränkungen der Öffentlichkeit des Verfahrens vgl. Grabenwarter/Pabel, in: Grote/Marauhn, (Fn. 75), Kap. 14, Rdnr. 111 ff.; Peukert, in: Frowein/ders., (Fn. 92), Art. 6, Rdnr. $191 \mathrm{ff}$. 
Pflichten aus Art. 6 Abs. 1 EMRK zu genügen, den Antrag eines Sportlers auf Durchführung eines öffentlichen Verfahrens nicht verweigern. ${ }^{124}$ Angesichts der in Sportlerkreisen teilweise vorherrschenden Skepsis gegenüber Entscheidungen von Sportschiedsgerichten, wie etwa die Unterstützung der eingangs skizzierten Petition Pechsteins zeigt, kann ein solches Antragsrecht schließlich auch der (Wieder-)Herstellung von Vertrauen in die Unabhängigkeit und Fairness der Entscheidungsfindung im Rahmen sportschiedsgerichtlicher Verfahren dienen. ${ }^{125}$

Soweit in der Literatur in dem Abschluss der Schiedsvereinbarung ein gleichzeitiger Verzicht auf weitere, essentielle Verfahrensrechte des Art. 6 Abs. 1 EMRK gesehen wird, ${ }^{126}$ muss dem widersprochen werden. Der (freiwillige) Verzicht auf Zugang zu einem staatlichen Gericht und auf die Öffentlichkeit des Verfahrens lässt sich noch regelmäßig auf den Willen der Parteien zurückführen; darauf dürfte sich die Reichweite des Verzichts aber beschränken. Denn auch vor einem Schiedsgericht erwarten die Parteien die Durchführung eines fairen Verfahrens durch ein unabhängiges und unparteiisches Gericht, welches innerhalb einer angemessenen Frist eine Entscheidung in der Sache trifft. Diese Erwartungen, so stellt Bangert ${ }^{127}$ zutreffend fest, korrespondieren mit den drei wesentlichen Inhalten des Art. 6 Abs. 1 EMRK: der Garantie eines unabhängigen und unparteiischen (Schieds-)Richters, der Garantie eines fairen Verfahrens mit umfassendem rechtlichen Gehör und der Garantie auf eine Entscheidung binnen angemessener Frist. ${ }^{128}$ Mit dem Abschluss einer Schiedsvereinbarung verzichten die Parteien mithin nicht auf diese essentiellen Verfahrensgrundrechte; unberührt hiervon verbleibt allerdings die Möglichkeit, auf diese Rechte während des anhängigen Prozesses zu verzichten.

\section{Sportspezifische Implikationen - zur „Freiwilligkeit“" der Schiedsvereinbarung im internationalen Sport und Art. 6 EMRK}

Für den wirksamen Verzicht von grundlegender Bedeutung ist letztlich die Freiwilligkeit der Schiedsvereinbarung. Diese von den Konventionsorganen wiederkehrend betonte Vor-

124 Den Antrag Pechsteins auf Zulassung ihres Managers im Verfahren vor dem CAS haben die Schiedsrichter aufgrund von R57 CAS-Code abgelehnt. Diese Weigerung hat Pechstein im noch anhängigen Verfahren vor dem EGMR (Nr. 67474/10, Pechstein/Suisse) als Verstoß gegen den Öffentlichkeitsgrundsatz gerügt. Nicht unwahrscheinlich ist es daher, dass der EGMR zu dem Ergebnis kommt, dass diese Weigerung eine Verletzung von Art. 6 Abs. 1 EMRK darstellt.

125 So auch BG v. 10.2.2010, 4A_612/2009, Rdnr. 4.1 in einem obiter dictum.

126 Vgl. etwa Jarrosson, (Fn. 8), S. 584, 589 f.; ähnlich Jacot-Guillarmod, (Fn. 14), S. 292; siehe ferner Ambrose, (Fn. 23), S. 484 mit Blick auf die Unabhängigkeit der Schiedsrichter. Insgesamt wohl auch großzügig in dieser Hinsicht Schultz, A Speed Bump for Arbitral Procedures?, An Exploration of Safeguards in the Acceleration of Justice, International Arbitration 2006, S. 13. Vgl. ferner Landrove, (Fn. 8), S. 85, der für die Zulässigkeit des Verzichts auf die Garantie der Entscheidung innerhalb einer angemessenen Frist plädiert.

127 Bangert, (Fn. 62), S. 50.

128 Ebenso Briner/Schlabrendorff, (Fn. 45), S. 94 ff.; Besson, (Fn. 17), S. 400; Matscher, (Fn. 50), S. 242 ff.; Robinson/Kasolowsky, (Fn. 14), S. 464; Knoepfler, (Fn. 8), S. 471 ff.; wohl auch Wedam-Lukic, (Fn. 8), S. 17, 19; Schiavetta, (Fn. 63), Rdnr. 4.1; Ambrose, (Fn. 23), S. 485; Kola-Tafaj, Implementation of the European Convention on Human Rights in Arbitration Proceedings, SchiedsVZ 2012, S. 188. 
aussetzung für einen wirksamen Verzicht ${ }^{129}$ wirft insbesondere im Zusammenhang mit der Sportschiedsgerichtsbarkeit Probleme auf, die einer näheren Untersuchung bedürfen. Dies gilt einmal mehr angesichts der gegenwärtigen Protestbekundungen einzelner Sportler im Hinblick auf die Zulässigkeit von Athletenvereinbarungen und der aktuellen Entscheidung des Landgerichts München, das in den dort niedergeschriebenen Schiedsvereinbarungen einen Verstoß gegen Art. 6 EMRK sieht. ${ }^{130}$

\section{Zur Praxis: Schiedsvereinbarungen im Sportrecht}

Die Freiwilligkeit der Schiedsvereinbarung ist gewöhnlich nicht weiter problematisch, resultiert - im Gegensatz zum staatlich angeordneten Schiedszwang - doch die Übereinkunft zur schiedsgerichtlichen Klärung aus dem privatautonomen Entschluss zur Derogation staatlicher Justiz. Nun kann es dennoch zu Situationen kommen, in denen es an Kräftegleichheit der Parteien mangelt. ${ }^{131}$ Im Sport entstehen derartige Ungleichgewichtslagen zwischen internationalem Verband und Sportler in der Regel im Zusammenhang mit sogenannten Regelanerkennungsverträgen bzw. Athletenvereinbarungen. ${ }^{132}$ Darin unterwerfen sich die Sportler den Regelwerken und der Disziplinargewalt der internationalen Sportverbände; in zunehmenden Maße ordnen diese Verbandsregularien auch den Ausschluss der staatlichen Gerichtsbarkeit an. So müssen etwa Teilnehmer an den Olympischen Spielen eine sogenannte entry-form unterzeichnen, die in Ziffer 8 eine Schiedsklausel enthält, wonach sämtliche Streitigkeiten, Kontroversen oder Schadensfälle, welche aus oder im Zusammenhang mit oder anlässlich der Olympischen Spiele hervorgehen, ausschließlich

129 Vgl. etwa EKMR, Nrn. 8588/79 und 8589/79, Bramelid and Malmström/Sweden; EKMR, Nr. 23173/94, Molin/Turquie; ebenso der EGMR, Nr.31737/96, Suovaniemi and others/Finland.

130 Siehe hierzu bereits die einleitenden Bemerkungen. Für eine verfassungsrechtliche Bewertung dieser Athletenvereinbarungen jüngst Zuck, Die DOSB-Athletenvereinbarung Sotschi 2014, verfassungsrechtlich betrachtet, SpuRt 2014, S. 5 ff.

131 Allgemein zu Ungleichgewichtslagen im deutschen Recht Schlosser, in: Stein/Jonas, (Fn. 94), $\S 1029$, Rdnr. 26; Lachmann, Handbuch Schiedsgerichtspraxis, 2002, Rdnr. 382 f.

132 Im Einzelnen Haas/Hauptmann, Schiedsvereinbarungen in Ungleichgewichtslagen - am Beispiel des Sports, SchiedsVZ 2004, S. 176 f.; Brinkmann, Öffentlich-rechtliche Gestaltung im Sportgerichtsverfahren?, 2006, S. 39 f.; Holla, Der Einsatz von Schiedsgerichten im organisierten Sport, 2006, S. 112 ff.; Andexer, Die Nationale Sportgerichtsbarkeit und ihre internationale Dimension, 2009, S. 288 ff. Jüngst mit Blick auf Sotschi Zuck, (Fn. 130), S. 5 f. 
dem CAS zur endgültigen und rechtsverbindlichen Entscheidung vorzubringen sind. ${ }^{133}$ Auch Art. 61 Abs. 2 der IOC-Charta sieht für Streitigkeiten, die im Zusammenhang mit den Olympischen Spielen auftreten, die ausdrückliche Zuständigkeit des CAS vor. Diese Praxis beschränkt sich aber nicht auf das IOC; auch andere internationale Sportverbandssatzungen beinhalten vergleichbare Klauseln, die Streitigkeiten an Schiedsgerichte verweisen. In diesen Konstellationen ist die durch die Formvorschrift induzierte Informationsparität nicht ausreichend für die Annahme der Freiwilligkeit der Schiedsabrede. Denn dem Sportler wird die Schiedsvereinbarung einseitig gestellt; er hat keinen Einfluss auf den Inhalt der Regelanerkennungsverträge und kann sich gleichzeitig aufgrund der monopolartigen Struktur internationaler Sportwettkämpfe der Vereinbarung insgesamt nicht entziehen.

\section{Rechtsprechungsübersicht}

Derartige ungleiche Kraftverhältnisse, die sich letztlich auf wirtschaftliche Notwendigkeit zurückführen lassen, führen allerdings nach Auffassung der Konventionsorgane nicht per se zur Unwirksamkeit der Schiedsabrede.

In der Rechtssache Axelsson and others/Sweden bestand gleichfalls eine wirtschaftliche Ungleichgewichtslage zwischen dem klagenden Taxifahrer und einem lokalen monopolistischen Taxiunternehmen. ${ }^{134}$ Dies nahm indes die Kommission nicht zum Anlass, die gegenständliche Schiedsvereinbarung wegen fehlender Freiwilligkeit für unwirksam zu erklären. ${ }^{135}$ Auch die standardmäßige Verwendung von Schiedsklauseln in Arbeitsverträgen vermochte die Kommission in der Rechtssache $X / R F D A^{136}$ nicht davon zu überzeugen, dass im konkreten Fall an der Freiwilligkeit zu zweifeln sei. In der Rechtssache Hedland/ Sweden ${ }^{137}$ war die Schiedsabrede nicht von dem Beschwerdeführer selbst vereinbart worden, sondern war Teil eines von einer schwedischen Gewerkschaft und einem privaten

133 Vgl. Art. 44, Durchführungsbestimmung Nr. 6 der Charta i.V.m. Ziffer 8 der „entry-form“ für Sotschi: „I agree that any dispute, controversy or claim arising out of, in connection with, or on the occasion of the Olympic Games, not resolved after exhaustion of the legal remedies established by my NOC, the International Federation governing my sport, Sochi 2014 and the IOC, shall be submitted exclusively to the Court of Arbitration for Sport ('CAS') for final and binding arbitration in accordance with the Arbitration Rules for the Olympic Games, which form part of the Code of Sports-related Arbitration. The arbitration shall be governed by Chapter 12 of the Swiss Act on Private International Law and its seat shall be in Lausanne, Switzerland. The CAS shall rule on its jurisdiction and shall have the exclusive power to order provisional and conservatory measures. The decisions of the CAS shall be final, binding and nonappealable. I shall not and I hereby waive my right to institute any claim, arbitration or litigation, or seek any other form of relief, in any other court or tribunal." Ohne die Unterzeichnung ist bereits eine Nominierung durch den DOSB nicht möglich, vgl. Beschluss des DOSB v. 18.9.2012 bzgl. der Grundsätze zur Nominierung für Sotschi. Zur Zulässigkeit dieser ,entry form" schon für Atlanta vgl. Netzle, in: Röhricht (Hrsg.), Sportgerichtsbarkeit, 1997, S. 17 f.; für Sydney Haas, (Fn. 20), S. 336 f.; für Sotschi Jakob-Milicia, Die „Entry-Form“ des IOC, SpuRt 2013, S. 236 ff. Zu dieser Form der Schiedsgerichtsbarkeit siehe ferner KaufmannKohler, Arbitration at the Olympics, 2001, S. $7 \mathrm{ff}$.

134 EKMR, Nr. 11960/86, Axelsson and others/Sweden.

135 Haas, (Fn. 8), S. 79; Samuel, (Fn. 19), S. 422 f.

136 EKMR, Nr. 1197/61, R/RFDA.

137 EKMR, Nr. 24118/94, Hedland/Sweden. 
Versicherungsunternehmen unterzeichneten „collective agreement“. Obwohl der Beschwerdeführer Mitglied der Gewerkschaft war, berief er sich auf eine Verletzung von Art. 6 Abs. 1 EMRK, da er seiner Ansicht nach nicht auf sein Recht auf Entscheidung durch ein staatliches Gericht freiwillig verzichtet habe. In ihrer Entscheidung musste die Kommission nicht näher auf die Frage der „Freiwilligkeit“ der Schiedsvereinbarung eingehen, da der Beschwerdeführer den Schiedsspruch zuvor nicht vor staatlichen Gerichten angefochten hatte. Wäre die Kommission indes der Ansicht, dass derartige Ungleichgewichtslagen im Lichte des Art. 6 Abs. 1 EMRK problematisch zu bewerten sind, hätte sie dies an dieser Stelle in einem obiter dictum ausdrücken können. Auch in der Rechtssache Molin/ Turquie monierte der Beschwerdeführer die fehlende Freiwilligkeit der Schiedsvereinbarung; die EMRK konnte aber ebenso wie in der Rechtssache Hedland/Sweden die nähere Untersuchung der Freiwilligkeit vermeiden, da der Kläger den innerstaatlichen Rechtsweg nicht ausgeschöpft hatte. ${ }^{138}$

Die Regelanerkennungsverträge in Sportverbandssatzungen lassen sich nun aber nur bedingt mit diesen von der EKMR entschiedenen Fällen vergleichen; dort verblieb den Klägern zumindest noch die Möglichkeit, ihren Beruf überhaupt auszuüben bzw. sich der Schiedsvereinbarung auf andere Art und Weise zu entziehen. Der Sportler hat im Gegensatz dazu de facto keine andere Wahl, als die Regelanerkennungsverträge zu unterzeichnen und damit auf sein Recht auf Entscheidung durch einen staatlichen Richter zu verzichten, will er mit sportlichen Leistungen seinen Lebensunterhalt erbringen. ${ }^{139}$ Demnach wirft diese Praxis im Lichte der Anforderung an die Freiwilligkeit der Schiedsabrede doch erhebliche Zweifel auf.

Derartige „Schiedszwänge“ in den Regularien internationaler Sportverbände sind bis dato noch nicht von den Konventionsorganen auf ihre Vereinbarkeit mit der EMRK überprüft worden. Sportverbandsrechtliche Normen, die einen Schiedszwang nahelegen, waren aber Gegenstand sowohl nationaler staatlicher Gerichts- als auch internationaler Schiedsverfahren. Erstaunlicherweise haben dabei aber weder der CAS noch der englische Court of Appeal die Freiwilligkeit der Schiedsvereinbarung im Rahmen von Anerkennungsverträgen überhaupt näher untersucht. Der CAS stellte in der Rechtssache Netherlands Antilles Olympic Committee/IAAF\&USOC lapidar fest, dass die Schiedsklausel die Klägerin binde, da sie die „IOC-entry form“ unterzeichnet habe, ohne auch nur die ungleichen Kräfteverhältnisse bei Abschluss der Schiedsvereinbarungen zu thematisieren. ${ }^{140}$ Der englische Court of Appeal untersuchte zumindest - im Übrigen mit Blick auf Art. 6 Abs. 1 EMRK die Wirksamkeit der Schiedsklausel, stellte aber ebenso apodiktisch fest, dass er konkret keine Zwangslage erblicken könne. ${ }^{141}$ Eingehender mit der Freiwilligkeit von Verzichtserklärungen im Allgemeinen setzte sich das Schweizer Bundesgericht in einem der wenigen

138 EKMR, Nr. 23173/94, Molin/Turquie.

139 Ebenso Adolphsen, Grundfragen und Perspektive der Sportschiedsgerichtsbarkeit, SchiedsVZ 2004, S. 172 f.; Haas, in: Gilles/Pfeiffer (Hrsg.), Neue Tendenzen im Prozessrecht, 2008, S. 40; Holla, (Fn. 132), S. 112.

140 Vgl. CAS, 2008/A/1641, Netherlands Antilles Olympic Committee/IAAF\&USOC, Rdnrn. 63, 65.

141 Court of Appeal v. 21.3.2007, APP.L.R. 03/21, Stretford v The Football Association and Another, Rdnr. 66: „No question of constraint here.“ Damit bestätigte der Court auch die vorangegangene Entscheidung des High Court; eingehend Grothe/Frohn, (Fn. 14), S. 104 ff. 
Verfahren auseinander, in dessen Verlauf letztlich ein Schiedsspruch des CAS aufgehoben wurde. Der Beschwerdeführer monierte eine Entscheidung des CAS, wonach dieser eine Verbandsnorm nicht beanstandet habe, obwohl diese neben der üblichen Schiedsvereinbarung zusätzlich auch einen Rechtsmittelverzicht bezüglich der Aufhebungsklage enthielt. In der Entscheidung heißt es zunächst, dass es

„offensichtlich [sei], dass der Rechtsmittelverzicht, der von einem Sportler erklärt wird, in aller Regel nicht seinem freien Willen entspricht". ${ }^{142}$

Aufgrund vorgestellter Ausführungen des Schweizer Bundesgerichts zur Schiedsklausel bezieht sich diese Aussage nicht nur auf die nach Art. 192 Abs. 1 IPRG mögliche Verzichtserklärung der Anfechtung von Schiedssprüchen, sondern auch auf die Schiedsvereinbarung, zumal das Bundesgericht in der Folge zwischen diesen beiden Formen differenziert. Allerdings hob das Gericht auch ausdrücklich hervor, dass hinsichtlich der Schiedsvereinbarung weitaus geringere Anforderungen an das Freiwilligkeitselement gestellt werden als bei einem Rechtsmittelverzicht, indem es auf das Interesse an einer raschen Erledigung von Streitigkeiten durch spezialisierte Sportschiedsgerichte hinweist. ${ }^{143} \mathrm{Im}$ Ergebnis erklärte das Bundesgericht daher auch nur den Rechtsmittelverzicht im Sinne des Art. 192 Abs. 1 IPRG für unwirksam, nicht aber auch die Schiedsvereinbarung. ${ }^{144}$

Die Diskussion hinsichtlich der Freiwilligkeit solcher Schiedsabreden im Sport hat mit einer aktuellen Entscheidung des Landgerichts München I neuen Auftrieb erhalten. ${ }^{145}$ Dort hatte das Gericht über eine Klage der deutschen Eischnellläuferin Claudia Pechstein gegen die Deutsche Eisschnelllauf-Gemeinschaft (DESG) und die International Skating Union (ISU) zu befinden, mit der die Klägerin Schadensersatz in Höhe von rund 4 Millionen Euro verlangt, weil eine gegen sie verhängte Dopingsperre rechtswidrig gewesen sein soll. ${ }^{146}$ Die Richter wiesen die Klage zwar im Ergebnis ab, weil sie sich hinsichtlich der Rechtmäßigkeit der Dopingsperre an das vorangegangene Urteil des CAS gebunden sahen. Allerdings erklärte sich das Gericht überhaupt für zuständig, weil es die Schiedsvereinbarungen zwischen den Sportverbänden und Pechstein für nicht rechtmäßig hielt. Dabei erkennt das Gericht nicht nur in der Schiedsvereinbarung zwischen Pechstein und der DESG einen Verstoß gegen $§ 138$ BGB, sondern erklärt darüber hinaus auch die Schiedsvereinbarung zwischen Pechstein und der ISU wegen der Verletzung von Art. 6 EMRK für unwirksam. Die Unwirksamkeit beider Schiedsabreden begründet das Gericht mit der strukturellen Unterlegenheit Pechsteins gegenüber den Sportverbänden beim Abschluss der Schiedsvereinbarung. Diese hatte bei der Unterzeichnung der Schiedsvereinbarungen keine Wahl; ohne die Unterzeichnung wäre die Klägerin nicht zu Wettkämpfen zugelassen worden und dadurch in ihrer Berufsausübung behindert gewesen. ${ }^{147}$

142 BG v. 22.3.2007, 4.P. 172/2006 = SchiedsVZ 2007, 330 (4.3.2.2.).

143 Ibid., 4.3.2.3.

144 Vgl. ibid., 4.4.2.

145 LG München I v. 26.2.2014, 37 O 28331/12.

146 Siehe die Pressemitteilung 03/14 v. 26.2.2014, Rechtsprechung des Landgerichts München I in Zivilsachen (Kein Schadensersatz für Eisschnellläuferin nach Dopingsperre).

147 Ibid. 


\section{Stellungnahme}

Auch wenn sich die Gerichte hinsichtlich der Frage der Freiwilligkeit solcher Schiedsabreden im internationalen Sport nicht einig sind, so muss im Lichte des Art. 6 Abs. 1 EMRK zunächst konstatiert werden, dass es in Anbetracht des oben skizzierten strukturellen Ungleichgewichts an der Freiwilligkeit der Schiedsabrede in sportspezifischen Fällen regelmäßig fehlen wird. ${ }^{148}$ Damit könnten derartige Schiedsabreden in Regelanerkennungsverträgen gegen Art. 6 Abs. 1 EMRK verstoßen, da das Recht auf Zugang zu einem staatlichen Gericht verletzt wäre. Dennoch bedeutet dies aus Sicht der EMRK nicht zugleich, dass der Schiedsvereinbarung ihre Wirksamkeit abgesprochen werden muss. ${ }^{149}$ Vielmehr ist das Recht auf Zugang zu einem (staatlichen) Gericht nur unter dem Vorbehalt verhältnismäBiger Einschränkungen gewährleistet. ${ }^{150}$ Derartige Einschränkungen sind zulässig, sofern sie dem Interesse einer ordnungsgemäßen Rechtspflege (,,bonne administration de la justice $)^{151}$ dienen wollen und den Wesensgehalt (,the very essence $)^{152}$ des Art. 6 Abs. 1 EMRK nicht verletzen. Davon kann in der Regel ausgegangen werden, sofern die Einschränkungen auf einer gesetzlichen Grundlage beruhen, ein legitimes Ziel verfolgen und verhältnismäßig sind. In diesem Sinne hat der EGMR mit Blick auf die gesetzlich angeordnete Schiedsgerichtsbarkeit entschieden, dass eine nationale Regelung, die darauf abzielt, eine Vielzahl von Individualklagen durch den Ausschluss der staatlichen sowie des schiedsgerichtlichen Rechtswegs zu verhindern, verhältnismäßig sein kann und den Wesensgehalt von Art. 6 Abs. 1 EMRK nicht zu berühren vermag. ${ }^{153}$ In dem Lichte dieses Urteils könnte folglich eine Schiedsvereinbarung auch dann als zulässig erachtet werden, wenn diese zwar auf gesetzlich angeordnetem Schiedszwang beruht, Gründe im Sinne einer ordnungsgemäßen Rechtspflege aber eine Beschränkung des Rechts auf Zugang zu einem staatlichen Gericht rechtfertigen.

148 Insgesamt auch für eine strenge Prüfung der „Freiwilligkeit“ der Unterwerfung vgl. Matscher, (Fn. 50), S. 243.

149 Im nationalen Recht enthielt § 1025 ZPO a.F. eine Regelung, wonach eine Schiedsvereinbarung unwirksam war, wenn eine Partei ihre Übermacht dazu ausnützt, den anderen Teil zum Abschluss zu nötigen; zur Zulässigkeit der Regelanerkennungsverträge i.R.d. § 1025 ZPO a.F. vgl. nur Haas/Holla, in: Haas (Hrsg.), Schiedsgerichtsbarkeit im Sport, 2003, S. 23 f. m.w.N. Eine vergleichbare Regelung sieht das Schiedsrecht nach der Reform des Schiedsverfahrensrechts nun nicht mehr vor. Damit entfällt aber nicht die Voraussetzung der Freiwilligkeit der Schiedsabrede. In einer noch zum alten Recht ergangenen Entscheidung des BGH stellte dieser für den Schutz der Freiwilligkeit nämlich nicht nur auf $\S 1025 \mathrm{ZPO}$ a.F., sondern auch auf verfassungsrechtliche Vorgaben ab, vgl. BGH v. 3.4.2000 = NJW 2000, 1713, Pkt. 2; kritisch Adolphsen, (Fn. 139), S. 175. Die h.M. verlangt dagegen, dass neben dem Bestehen einer Ungleichgewichtslage besondere Umstände hinzutreten, die eine Sittenwidrigkeit begründen, damit die Wirksamkeit der Schiedsabrede verneint werden kann, vgl. nur Haas/Reiche, Aufhebung eines Schiedsurteils des CAS, SchiedsVZ 2007, S. 336 m.w.N.; a.A. indes Monheim, Die Freiwilligkeit von Schiedsabreden im Sport und das Rechtsstaatsprinzip, SpuRt 2008, S. 9 f.

150 Vgl. hierzu Grabenwarter/Pabel, (Fn. 11), §24, Rdnr. 49 ff.; Peukert, (Fn. 123), Art. 6, Rdnr. 64 ff.; Grabenwarter/Pabel, (Fn. 123), Kap. 14, Rdnr. 71 ff. Siehe ferner Matscher, in: Festschrift Henckel, (Fn. 23), S. 599.

151 EKMR, Nr. 6916/75, X.Y.Z./Suisse, D.R. 6, S. 109.

152 EGMR, Nr. 8225/78, Ashingdane/UK, Rdnr. 57; zuletzt EGMR, Nr. 8917/05, Kart/Turkey, Rdnr. 79.

153 EGMR, Nr. 9006/80, Lithgow and others/UK, Rdnr. $196 \mathrm{f}$. 
Sind unter diesen Umständen staatliche Einschränkungen des Rechts auf Zugang zu einem ordentlichen Gericht mit der Konvention vereinbar, so schließt sich die Frage an, ob auch sportverbandliche Regelungen dieses Recht in zulässiger Weise beschränken können. Aus Sicht der EMRK ist es zunächst unerheblich, ob die Schiedsvereinbarung von staatlicher Seite oktroyiert wird oder ob der Schiedszwang auf private Initiative zurückgeht; entscheidend ist einzig die Verkürzung von Konventionsrechten. Befürwortet der EGMR aber die prinzipielle Möglichkeit der Abwägung des Rechts auf Zugang zu einem Gericht bei der gesetzlich angeordneten Schiedsgerichtsbarkeit, dann spricht einiges dafür, diesen Grundsatz auch auf monopolistische Sportverbandsnormen zu übertragen. ${ }^{154}$ Denn der Sportverband ist in diesen Fällen in funktioneller Hinsicht nichts anderes als eine Art „Quasigesetzgeber“ im Hinblick auf die Sportler. Auch der internationale Sportverband verfolgt nämlich nicht in erster Linie, wie dies etwa sonst Privatrechtssubjekten eigen ist, selbstnützige Ziele, sondern übergeordnete, im Allgemeininteresse aller Sportler liegende Zwecke. ${ }^{155}$

Anknüpfungspunkt einer Überprüfung durch den EGMR ist daher die Tatsache, dass eine staatliche Rechtsordnung derartige „Kontrahierungszwänge“ überhaupt billigt. Um die Konventionswidrigkeit zu überprüfen, bietet es sich aus der Sicht der EMRK an, die Anforderungen an zulässige, staatliche Beschränkungen des Zugangs zu einem ordentlichen Gericht auf gleichwertige Begrenzungen durch ,quasi-gesetzgeberisch“ tätige Sportverbände zu übertragen, wobei dies - wie voranstehend angeführt - nicht bedeutet, dass die Sportverbände etwa an Art. 6 Abs. 1 EMRK gebunden wären. Ansetzen kann der EGMR nur an der staatlichen Billigung des Schiedszwangs durch die der Konvention verpflichteten Staaten. Damit ergeben sich für zulässige Beschränkungen folgende Voraussetzungen: a) eine „gesetzliche“ Grundlage des Schiedszwangs, b) ein legitimes Ziel und c) Verhältnismäßigkeit.

Für die Rechtfertigung einer Beschränkung ist also grundsätzlich zunächst eine (gesetzliche) Grundlage erforderlich. ${ }^{156}$ Mit Blick auf die Schiedsvereinbarung findet sich diese in der Regel in der internationalen Dachverbandssatzung oder in den Verfahrensregeln internationaler Sportgerichte. Nun fordert der EGMR im Rahmen staatlicher Eingriffe, dass die gesetzliche Grundlage vorsehbar, also zugänglich und hinreichend bestimmt ist. ${ }^{157}$ Mit Blick auf die Satzungen und Verfahrensregeln folgt daraus, dass die Sportverbände und -institutionen gewährleisten müssen, dass die jeweilige Schiedsvereinbarung den Athleten in irgendeiner Form zugänglich ist. ${ }^{158}$

Weiterhin muss die Beschränkung des Art. 6 Abs. 1 EMRK ein legitimes Ziel verfolgen. Ein solches Ziel lässt sich in der Einheitlichkeit der Sportausübung erblicken. ${ }^{159}$ Vergleichbare Wettbewerbsbedingungen sind ein Wesensmerkmal des mittlerweile globali-

154 Vgl. auch Haas, (Fn. 8), S. 80; etwas knapp Grothe/Frohn, (Fn. 14), S. 109.

155 Dazu sogleich.

156 Eingehend Grabenwarter/Marauhn, in: Grote/Marauhn, (Fn. 75), Kap. 7, Rdnr. $21 \mathrm{ff}$.

157 Vgl. nur EGMR, Nr. 6538/74, Sunday Times, Rdnr. 49; EGMR, Nr. 5947/72, Silver and others/ UK, Rdnr. 85 ff. Eingehend Grabenwarter/Marauhn, (Fn. 156), Kap. 7, Rdnr. 27 ff.

158 Schultz, (Fn. 126), S. 15.

159 Statt vieler Haas, (Fn. 139), S. 13 ff. m.w.N. 
sierten Sports. ${ }^{160}$ Vor diesem Hintergrund gebietet die Internationalität des Sports, dass die Wettbewerbe und Wettkämpfe nach einheitlichen Regularien ausgetragen werden. Chancengleichheit und Vergleichbarkeit der Wettkampfleistungen lassen sich nur dann gewährleisten, wenn auch die Sanktionshoheit über die festgesetzten Regelwerke in einheitlicher Weise ausgeübt wird. Auf Grund der Zuständigkeit staatlicher Gerichte sind die internationalen Verbände an der gleichmäßigen Durchsetzung der von ihnen verhängten Sanktionen dann gehindert, wenn die Sportler von ihrem Recht Gebrauch machen, Rechtsschutz vor staatlichen Gerichten zu suchen. ${ }^{161}$ Dies führt zwangsläufig zu divergierenden Entscheidungen, da das nationale Recht unterschiedliche Schutzmechanismen vorsieht; auch ist der staatliche Richter zwar wohl berechtigt, keinesfalls aber verpflichtet, der globalen Dimension des Sports Rechnung zu tragen. Angesichts der weltweiten Gerichtspflichtigkeit internationaler Sportverbände ${ }^{162}$ droht die „Zersplitterung“ des internationalen Sportrechts. ${ }^{163}$ Eine denkbare Lösung dieses „Dilemmas“164 ist daher im Prozessrecht zu finden; internationale Sportschiedsgerichte können vor diesem Hintergrund zur Rechtseinheit im internationalen Sport entscheidend beitragen. ${ }^{165}$

Letztlich muss die Beschränkung des Zugangs zum (staatlichen) Gericht verhältnismäßig sein. Das Interesse der internationalen Sportverbände an einheitlicher Rechtsdurchsetzung muss folglich das Recht des Sportlers auf Zugang zu einem staatlichen Gericht überwiegen. $\mathrm{Zu}$ berücksichtigen bei der Abwägung ist, dass die Zuständigkeitskonzentration an internationalen Schiedsgerichten auch den Sportlern selbst dient. Denn auch diese sind an der einheitlichen Rechtsdurchsetzung der sportverbandsrechtlichen Regeln interessiert, haben sie sich doch den Regelanerkennungsverträgen nur unter der Voraussetzung der Gleichbehandlung aller beteiligten Athleten unterworfen. ${ }^{166}$ Die Geltung einheitlicher Rechtsregeln ist demnach ein Anliegen von gemeinsamem Interesse sowohl der Verbände

160 Vgl. Ronellenfitsch, Die Zulassung von Automobilsportveranstaltungen, DAR 1995, S. 241.

161 Adolphsen, (Fn. 139), S. 170.

162 Zur Problematik des sog. forum shoppings in diesem Zusammenhang vgl. Schleiter, Globalisierung im Sport, 2009, S. 20 f.; Kotzenberg, Bindung des Sportlers an private Dopingregeln und private Schiedsgerichte, 2007, S. $156 \mathrm{f}$.

163 Vgl. auch Vieweg, Normsetzung und -anwendung deutscher und internationaler Verbände, 1990, S. $187 \mathrm{f}$; Vedder, The International Olympic Committee: An Advanced NON-Governmental Organization and the International Law, GYIL 27 (1984), S. 235; Bach, Der Dopingfall Harry „Butch“ Reynolds - Plädoyer für internationale Sportgerichtsbarkeit, SpuRt 1995, S. 142; Reuter, Das selbstgeschaffene Recht des internationalen Sports im Konflikt mit dem Geltungsanspruch des nationalen Rechts, DZWir 1996, S. 1 f.; Weller, Die FIFA-Fußball-WM im Lichte des Privatrechts, JuS 2006, S. 499; Wax, Internationales Sportrecht, 2009, S. 131 ff. Eingehend zu Defiziten der Streitbeilegung durch staatliche Gerichte Holla, (Fn. 132), S. 29 ff.

164 Adolphsen, (Fn. 139), S. 170.

165 Dafür plädieren auch Adolphsen, Internationale Dopingstrafen, 2003, S. 484; Haas, in: Führungs- und Verwaltungsakademie des Deutschen Sportbunds (Hrsg.), Akademieschrift Nr. 49, 1997, S. 68; Netzle, Jurisdiction of arbitral tribunals in sports matters: arbitration agreements incorporated by reference to regulations of sports-organisations, ASA Bulletin 1998, S. 47; Seitz, Hexenjagd auf Dopingsünder - Ein bundeseinheitliches Schiedsgericht für Sportdopingsachen muss her!, NJW 2002, S. 2838 f.; Bach, (Fn. 163), S. 143. Zu weiteren Lösungsansätzen dieses „Dilemmas“ vgl. Wax, (Fn. 163), S. 135 ff.; Schleiter, (Fn. 162), passim.

166 BGH, NJW 1995, 583 (584). Vgl. auch Pfister, in: Fritzweiler/Pfister/Summerer, Praxishandbuch Sportrecht, 2. Aufl. 2006, Kap. 6, Rdnr. 150; Haas, (Fn. 8), S. 81; Steiner, Verhältnis von Schiedsgerichtsbarkeit und staatlicher Gerichtsbarkeit, SpuRt 2014, S. 4. 
als auch der Sportler. Für die Verhältnismäßigkeit derartiger Schiedsvereinbarungen spricht in diesem Zusammenhang auch, dass der Sportler nicht gänzlich rechtslos gestellt wird. Es verbleibt die Möglichkeit der Anrufung spezialisierter Sportschiedsgerichte, deren Entscheidung in qualitativer Hinsicht den Urteilen staatlicher Gerichte in der Regel nicht nachstehen. Auch die Schnelligkeit der Entscheidungsfindung im Rahmen sportschiedsgerichtlicher Verfahren dient dem Sportler, da sich Verzögerungen ungünstig auf Teilnahmemöglichkeiten auswirken können.

Unter vorstehenden Voraussetzungen ist diese Form der Schiedsvereinbarung im Rahmen von Regelanerkennungsverträgen bzw. Athletenvereinbarungen deshalb mit Art. 6 Abs. 1 EMRK vereinbar. ${ }^{167}$

\section{E. Fazit}

Das Recht auf ein ,fair trial“ ist ein Menschenrecht erster Güte. Dies zeigt nicht nur die Bedeutung des Art. 6 Abs. 1 EMRK, sondern auch die Kodifikation vergleichbarer Verfahrensrechte in anderen völkerrechtlichen Abkommen. ${ }^{168}$ Die Gewährleistung von Verfahrensgrundrechten gilt freilich mit Blick auf schiedsgerichtliche Entscheidungen nur in eingeschränktem Umfang, macht man mit der Privatautonomie, ebenfalls verfassungs- und völkerrechtlich garantiert, ernst. Ziel muss es daher sein, einen angemessenen Ausgleich im Spannungsverhältnis der beiden elementaren Menschen- und Grundrechtspositionen zu finden. Der weitgehende Verzicht der Konventionsorgane wird der Aufgabe indes nicht gerecht, nehmen doch EKMR und EGMR eine Prüfung der Konventionsvereinbarkeit von Schiedsentscheidungen nur in dem Maße vor, wie das nationale Recht dahingehende Regelungen überhaupt bereithält. Durch den Abschluss der Schiedsvereinbarung wollen die Parteien in aller Regel aber nicht auf die essentiellen Verfahrensrechte des Art. 6 Abs. 1 EMRK verzichten. Deswegen bedarf die Rechtsprechung der Konventionsorgane einer Korrektur. Ob dies über eine unmittelbare Bindung der Schiedsgerichte an Art. 6 Abs. 1 EMRK bewerkstelligt wird oder aber über den Umweg einer positiven Schutzpflicht ist wohl nicht von allzu großer Bedeutung; ausschlaggebend ist die stärkere Einbeziehung von Schiedssprüchen in den Anwendungsbereich der EMRK. Angesichts einiger Ungereimtheiten der Theorie der mittelbaren Drittwirkung und der Zurechenbarkeit von Schiedsentscheidungen an den Konventionsstaat liegt es nahe, auch die Sportschiedsgerichte als unmittelbare Adressaten des Art. 6 Abs. 1 EMRK zu sehen. Gleichzeitig wird aber auch den Besonderheiten schiedsgerichtlicher Verfahren Rechnung getragen, denn es verbleibt aus Sicht der Konvention den Parteien unbenommen, auf eine Reihe von Verfahrensrechten durch den Abschluss einer Schiedsvereinbarung zu verzichten. Daher ist es aus Sicht der Konvention auch nicht zu beanstanden, wenn die Athleten für die Zulassung zu Wettkämpfen Vereinbarungen unterzeichnen müssen, worin die Zuständigkeit eines Internationalen Sportschiedsgerichts, etwa des CAS, als letzte Instanz in Streit- und Dopingfällen bestimmt wird. Zwar ist angesichts des wohl häufig fehlenden Freiwilligkeitselement grundsätzlich eine Berührung des in Art. 6 Abs. 1 EMRK garantierten Rechts auf Zugang

167 Im Ergebnis auch Grothe/Frohn, (Fn. 14), S. 109; Haas, (Fn. 8), S. 81; Zuck, (Fn. 130), S. 9 f.

168 Vgl. etwa Art. 14 IPbpR; Art. 8 AMRK. 
zu einem Gericht denkbar. Allerdings sprechen im Ergebnis die besseren Gründe dafür, eine solche Beschränkung aufgrund des Interesses aller Beteiligten an der Einheitlichkeit der Sportausübung nicht als Verletzung von Art. 6 Abs. 1 EMRK aufzufassen.

Die Tatsache, dass Schiedsgerichte an Art. 6 Abs. 1 EMRK gebunden sind, kann im Übrigen auch der Weiterentwicklung der Sportschiedsgerichtsbarkeit dienen. So kann der EGMR dazu beitragen, gewisse prozessuale Mindestbedingungen für schiedsgerichtliche Verfahren zu formulieren, die im gesamten Rechtsraum der EMRK gelten. Angesichts zumeist fehlender Rechtsprechungsübersichten im sportschiedsgerichtlichen Verfahren sowie divergierender staatlicher Überprüfungsmechanismen kann der EGMR „Richtlinien“ für die Ausgestaltung schiedsgerichtlicher Verfahren aufstellen. Letztlich wird diese Annahme auch nicht zu einer Flut von Beschwerdeverfahren führen, was angesichts gegenwärtig schon bestehender Arbeitsüberlastung des EGMR befürchtet werden könnte. Denn es bleibt den Staaten letztlich unbenommen, Verletzungen von Art. 6 Abs. 1 EMRK durch entsprechende legislative und judikative Schutzvorkehrungen zu vermeiden. Ganz der subsidiären Funktion der Konvention entsprechend soll es Aufgabe der Konventionsstaaten bleiben, Verletzungen von Art. 6 Abs. 1 EMRK in schiedsgerichtlichen Verfahren vorrangig abzuhelfen. 